\title{
Large-Eddy Simulation of Spray Flames in the DLR Generic Single Sector Combustor
}

\author{
Florian Setzwein, Peter Ess ${ }^{\dagger}$ and Peter Gerlinger ${ }^{\ddagger}$ \\ Institute of Combustion Technology, German Aerospace Center (DLR), Stuttgart, 70569, \\ Germany
}

\begin{abstract}
The lean burn combustion concept has shown potential for a further reduction of pollutants from aero engines. Partially premixed swirled flames in lean burn combustors strongly influence stability, performance and pollutant emissions associated with this concept and remain a major challenge when it comes to their prediction by numerical simulations. Large-Eddy Simulations (LES) for non-reactive and chemically reactive two-phase flows are performed for the DLR Generic Single Sector Combustor, which is a generic spray burner typical for lean burn combustors. Most simulations published on this kerosene burner do not accurately capture the lift-off height of the flame and the temperature distributions within the flow field. In contrast to previous scale-resolving simulations, the focus of this work is on LES, employing Finite-Rate Chemistry (FRC) for detailed combustion modeling. A reaction mechanism with 59 species and 372 elementary reactions and an Assumed Probability Density Function (APDF) approach for turbulence-chemistry interaction is used. The non-reacting mean velocity field, the spray distribution, flame shape, and flame lift-off height obtained from the simulations agree well with experimental data. In the flame, both premixed and diffusion-dominated regions are identified. Premixed zones occur predominantely, mainly as coherent structures in areas with high temperature gradients, whereas diffusion-dominated flame zones occur as isolated regions. For the detailed analysis of the burner, the employed numerical framework, including FRC, was found to be essential for an accurate prediction of the complex flame structure.
\end{abstract}

\section{Introduction}

Reducing emissions of civil aero engines has been a key objective in the last decades and will gain importance for the design of next generation engines, since more stringent regulations for aircraft emissions will come into effect. For instance, a milestone of the European Comission's Flightpath 2050 is the reduction of $\mathrm{NO}_{x}$ emissions by $90 \%$ towards the capabilities of typical new aircrafts from the year $2000 !$ Lean burn combustion concepts offer a high potential for the reduction of pollutant emissions due to lower flame temperatures and have been in the focus of many research projects. ${ }^{[2]}$ The overall stability and performance of such lean combustors in gas turbines depend on specific demands, for example the mixing of the hot exhaust gas with unburned reactants through recirculation. Computational Fluid Dynamics (CFD) can thereby act as a powerful tool in the design process and reduce the amount of experimental testing required ${ }^{2}$ Due to lower computational costs, Reynolds-Averaged Navier-Stokes (RANS) simulations are often employed during the design stage. Hereby, mean values of flow quantities are obtained through the solution of the temporally averaged Navier-Stokes equations and the effect of fluctuations on the mean flow is described through casespecific turbulence models. However, this approach can be insufficient in resolving highly complex flows, as they occur in modern lean combustors ${ }^{3}$ LES can overcome the problems of Reynolds-averaged simulations, since energy-rich, inhomogenous structures are resolved directly. This is obtained through the application of a spatial filtering operation on the governing equations, which decomposes the flow quantities into a sum of resolved and unresolved components $\underline{4}$ Only effects of small scale flow features are modeled, which have

*PhD Student, German Aerospace Center (DLR), Stuttgart, 70569, Germany

$\dagger$ Postdoctoral Fellow, German Aerospace Center (DLR), Stuttgart, 70569, Germany, AIAA Senior Member

$\ddagger$ Professor, German Aerospace Center (DLR), Stuttgart, 70569, Germany 
a more universal influence on the mean flow $\stackrel{5}{5}$ Thus, LES are more accurate than RANS computations for turbulent flows with large-scale unsteady motions. ${ }^{4}$ This has a big impact on simulations of turbulent flames in lean burn combustors, since the turbulent transport of reactive species has a major influence on the overall flow field. LES are therefore a common choice for simulating premixed and non-premixed combustion applications. As liquid fuels are used in aero engines, further complexity is introduced due to the presence of dispersed fuel droplets. Such two-phase flows can be modeled in detail using approaches such as the Volume of Fluids (VOF) method or Smoothed Particle Hydrodynamics (SPH). However, these methods require a large computational effort. A further approach is the Eulerian-Lagrangian coupling of the gaseous phase and the dispersed liquid phase. In this approach droplets are represented by mathematical parcels and processes like dispersion, evaporation and droplet interactions are included in an efficient manner. The gaseous phase is solved in an Eulerian framework, for which established CFD methods can be used. This offers the possibility to provide highly accurate solutions, while requiring less computational effort than the mentioned methods.

Based on the Eulerian-Lagrangian approach, LES are performed for the DLR Generic Single Sector Combustor within this work. Other groups have already carried out numerical simulations on this burner.6 ${ }^{[9}$ However, important features such as the lift-off height of the flame and temperature peaks within the averaged flow field could not be captured, even though various combustion models were employed. Jones et al. ${ }^{6}$ used a global mechanism for kerosene combustion and could reproduce experimental data for Sauter Mean Diameters (SMD) and velocity distributions of droplets very accurately. It was pointed out, that a complex flame structure occurs within this burner, with a coexistence of premixed and non-premixed regions. This was confirmed in the work of Andreini et al.,7 where LES were performed with the Flamelet Generated Manifold (FGM) approach for combustion modeling. The FGM method led to errors in the temperature and spray distribution, since a leading premixed behaviour was predicted, even in non-premixed regions. Puggelli et al ${ }^{9}$ performed different simulations with the FGM approach, where a detailed reaction mechanism of 96 species and 856 reactions was employed, and the Artificially Thickened Flame (ATF) model with a two-step global mechanism. In both cases the flame height has been overestimated and it was highlighted that the spray boundary condtions play a major role on the flame stabilization process.

The focus of this work lies in the assessment of scale-resolved simulations, together with the FRC approach for combustion modeling. For every species a transport equation is solved. For reasons of computational feasibility, a reduced chemical mechanism for kerosene combustion is used in the LES, which involves 59 species and 372 elementary reactions. The aim of this work is to obtain an improved prediction of flame characteristics through the application of FRC. Simulations are performed for a non-reacting and reacting operating point at elevated pressure. Both operating points are validated against experimental measurements. Furthermore, the detailed results of the employed approach are used to characterize in depth the occurence of different flame regimes in the combustion chamber.

\section{The DLR Generic Single Sector Combustor}

The DLR Generic Single Sector Combustor has been developed within the framework of the TIMECOPAE project. This burner has been designed to generate spray-conditions of typical aero engine burners, while preserving well defined boundary conditions for numerical simulations ${ }^{10}$ It consists of a co-rotating double radial swirler, which is fed by preheated air from a cylindrical plenum. Additional air is used for cooling walls and windows to facilitate diagnostic measurements. Kerosene is injected through a pre-filming air blast atomizer, where the liquid film is produced through an annular slot, as depicted in Figure 1 1 The combustion chamber features a length of $264 \mathrm{~mm}$ and a rectangular cross-sectional area of $102 \times 102 \mathrm{~mm}^{2}$. The burner was investigated experimentally in the work of Freitag et al! 11 and Meier et al! 10 Three different operating points were examined, which are shown in Table 1. In this work, only the operating points A and $\mathrm{C}$ are considered, with an operating pressure of four bar. Laser Doppler Anemometry (LDA) was used for measuring velocity profiles in the non-reacting flow field. For both reacting operating points, Phase Doppler Anemometry (PDA) was utilized for the analyis of spray features, such as droplet velocities, SMD and volume fluxes. Planar Laser Induced Fluorescence (PLIF) measurements of kerosene were employed for the visualization of vapor and the liquid fuel phase, as well as planar Mie scattering for the imaging of the liquid fuel phase. Temperature distributions in lean regions of the flame were determined with PLIF measurements of $\mathrm{OH}$. The visualization of reaction zones within the flow field was realized through $\mathrm{OH}$ chemiluminescence by Abel inversion. The visualization of reaction zones within the flow field was realized through $\mathrm{OH}$ chemiluminescence by Abel inversion. 


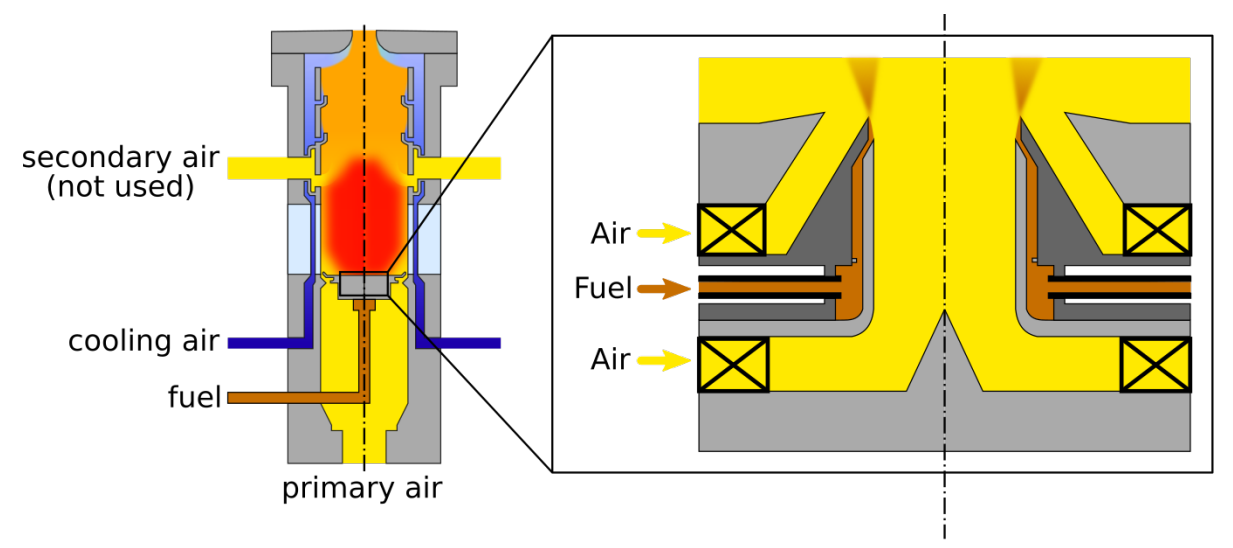

Figure 1: Schematics of the DLR Generic Single Sector Combustor. Adapted from Freitag et al!11

Table 1: Experimentally examined operating points of the DLR Generic Single Sector Combustor.

\begin{tabular}{lcccc}
\hline Operating point & Inlet air pressure & Air temperature & Air massflow-rate & Fuel massflow-rate \\
\hline A & $4 \mathrm{bar}$ & $550 \mathrm{~K}$ & $60 \mathrm{~g} / \mathrm{s}$ & $3.0 \mathrm{~g} / \mathrm{s}$ \\
$\mathrm{B}$ & $10 \mathrm{bar}$ & $650 \mathrm{~K}$ & $140 \mathrm{~g} / \mathrm{s}$ & $6.8 \mathrm{~g} / \mathrm{s}$ \\
$\mathrm{C}$ & $4 \mathrm{bar}$ & $295 \mathrm{~K}$ & $82 \mathrm{~g} / \mathrm{s}$ & $0.0 \mathrm{~g} / \mathrm{s}$ \\
\hline
\end{tabular}

\section{Numerical Modeling}

The simulation of the gasous phase is performed with the DLR in-house code Turbulent Heat Release Extension of the TAU Code (THETA) ${ }^{[12}$ whereas for the disperse phase, the DLR in-house code SPRAYSIM ${ }^{13}$ is employed. The following section describes the utilized LES modeling approaches for the gaseous and the disperse phase.

\section{A. Modeling of the Gaseous Phase}

Starting point for LES is the separation of any quantity $\Psi(\mathbf{x}, t)$ within the flow field into large scales and unresolved, subgrid-scales by utilization of a spatial filter operation. ${ }^{5}$ The spatial filtering is denoted by an overbar $\bar{\Psi}(\mathbf{x}, t)$. A Favre filtering $\widetilde{\Psi}=\overline{\rho_{g} \Psi} / \bar{\rho}_{g}$ with the gas density $\rho_{g}$ is employed to treat high density variations due to large temperature changes within the flow 14 Applying the density-weighted filter operation to the Navier-Stokes equations leads to

$$
\begin{aligned}
\frac{\partial \bar{\rho}_{g}}{\partial t}+\frac{\partial}{\partial x_{i}}\left(\bar{\rho}_{g} \widetilde{u}_{i}\right) & =\bar{S}_{\rho}^{d} \\
\frac{\partial}{\partial t}\left(\bar{\rho}_{g} \widetilde{u}_{i}\right)+\frac{\partial}{\partial x_{j}}\left(\bar{\rho}_{g} \widetilde{u}_{i} \widetilde{u}_{j}\right) & =-\frac{\partial}{\partial x_{j}}\left(\bar{\tau}_{i j}-\tau_{i j}^{S G S}\right)-\frac{\partial \bar{p}}{\partial x_{i}}+\bar{\rho}_{g} f_{i}+\bar{S}_{\rho u_{i}}^{d} \\
\frac{\partial}{\partial t}\left(\bar{\rho}_{g} \widetilde{h}\right)+\frac{\partial}{\partial x_{i}}\left(\bar{\rho}_{g} \widetilde{u}_{i} \widetilde{h}\right) & =-\frac{\partial}{\partial x_{i}}\left(\bar{q}_{i}-q_{i}^{S G S}\right)+\frac{\partial \bar{p}}{\partial t}+\bar{\rho}_{g} f_{i} \widetilde{u}_{i}+\bar{S}_{h}^{d} \\
\frac{\partial}{\partial t}\left(\bar{\rho}_{g} \widetilde{Y}_{\alpha}\right)+\frac{\partial}{\partial x_{i}}\left(\bar{\rho}_{g} \widetilde{u}_{i} \widetilde{Y}_{\alpha}\right) & =-\frac{\partial}{\partial x_{i}}\left(\bar{j}_{Y_{\alpha}, i}-j_{Y_{\alpha}, i}^{S G S}\right)+\bar{S}_{Y_{\alpha}}^{\mathrm{reac}}+\bar{S}_{Y_{\alpha}}^{d} .
\end{aligned}
$$

The filtered Navier-Stokes equations are solved for the velocity vector $\widetilde{\mathbf{u}}$, the pressure $\bar{p}$, the enthalpy $\widetilde{h}$ and species mass fractions $\widetilde{\mathbf{Y}}_{\alpha}$. The terms $\bar{S}_{i}^{d}$ denote source terms due to the presence of droplets of the disperse phase. External forces, such as gravitation, are represented by the force vector $f_{i}$. The terms $\tau_{i j}, q_{i}$ and $j_{i}$ refer to the viscous stress tensor, the energy flux and the species diffusion fluxes, respectively, and are split into filtered and unresolved components. Chemical source terms are denoted by $\bar{S}_{Y_{\alpha}}^{\text {reac }}$. The equations are solved by means of DLR's in-house code THETA, which is a 3D finite-volume solver for incompressible flows 
on unstructured median-dual grids. The filtering operation is implicitly associated with the finite-volume discretization, leading to a filter width $\Delta=(\Delta x \Delta y \Delta z)^{1 / 3}$, which is directly related to the computational grid width $\Delta x_{i}$. The pressure-velocity coupling, which arises from the incompressiblity constraint, is handled using a fractional step approach. A Poisson equation is solved for the pressure correction through the Flexible Generalized Minimal Residual Method (FGMRES) with a single multigrid V-cylce for convergence acceleration. Convective terms are discretized through a second-order accurate scheme with low-dissipation and low-dispersion properties, which has been described by Löwe et al 15 and tested for complex 3D flows by Probst et al 16 For the temporal discretization a second-order Three-Point Backward (TPB) scheme is employed. The enthalpy and temperature of the gaseous mixture are related by

$$
h=\sum_{\alpha=1}^{N_{s p}} h_{\alpha} Y_{\alpha} \quad \text { with } \quad h_{\alpha}=\Delta h_{f, \alpha}^{0}+\int_{T_{0}}^{T} c_{p, \alpha} \mathrm{d} T
$$

with the specific heat capacity at constant pressure $c_{p, \alpha}$ of species $\alpha$ and its standard enthalpy of formation $h_{f, \alpha}^{0}$. The gas density is calculated by the ideal gas law

$$
\rho_{g}=\frac{p}{R_{u} T \sum_{\alpha=1}^{N_{s p}} \frac{Y_{\alpha}}{M_{\alpha}}}
$$

with the universal gas constant $R_{u}$ and the molecular weight $M_{\alpha}$ of species $\alpha$. The filtered viscous stress tensor $\widetilde{\tau}_{i j}$ in equation $\sqrt{1 \mathrm{~b}}$ is assumed to be linearly dependent on the strain-rate through the kinematic viscosity $\nu \frac{17}{17}$ and is calculated using

$$
\widetilde{\tau}_{i j}=-\bar{\rho}_{g} \nu\left[\frac{\partial \widetilde{u}_{i}}{\partial x_{j}}+\frac{\partial \widetilde{u}_{j}}{\partial x_{i}}-\frac{2}{3} \delta_{i j} \frac{\partial \widetilde{u}_{k}}{\partial x_{k}}\right],
$$

where $\delta_{i j}$ is the Dirac delta function. Since low Mach number flows are considered, viscous dissipation terms $\tau_{i j} \partial u_{i} / \partial x_{j}$ are omitted in the enthalpy-equation $1 \mathrm{c}$ and the substantial derivative of pressure is approximated by $\mathrm{D} p / \mathrm{d} t=\partial \bar{p} / \partial t^{18}$. Thermal conduction and energy fluxes due to species diffusion are considered, whereas radiative heat losses are neglected. Therefore the filtered energy flux $\bar{q}_{i}$ in equation (1c) is calculated using

$$
\bar{q}_{i}=-\bar{\lambda} \frac{\partial \widetilde{T}}{\partial x_{i}}+\sum_{\alpha=1}^{N_{s p}} \widetilde{h}_{\alpha} \bar{j}_{Y_{\alpha}, i}
$$

with the filtered thermal conductivity $\bar{\lambda}$. The filtered species diffusion fluxes $\bar{j}_{Y_{\alpha}, i}$ are approximated through Fick's law, neglecting diffusion due to temperature and pressure gradients

$$
\bar{j}_{Y_{\alpha}, i}=-\bar{\rho}_{g} \bar{D}_{\alpha} \frac{\partial \widetilde{Y}_{\alpha}}{\partial x_{i}}
$$

The unresolved subgrid Reynolds stress tensor $\tau_{i j}^{s g s}$ in the momentum equations $1 \mathrm{~b}$ is modeled through the Wall-Adapting Local Eddy-Viscosity (WALE) model 1920 In the sense of classical RANS approaches, a turbulent eddy viscosity $\nu_{t}$ is introduced to model viscous effects of the smallest flow-structures on the resolved flow. The unresolved fluxes $q_{i}^{s g s}$ and $j_{Y_{\alpha}, i}^{s g s}$ are modelled by a gradient-diffusion approach, with the turbulent Prandtl number $\operatorname{Pr}_{t}=0.7$ and the turbulent Schmidt number $\mathrm{Sc}_{t}=0.7$.

$$
\begin{aligned}
\tau_{i j}^{s g s} & =\bar{\rho}_{g} \nu_{t}\left(\frac{\partial \widetilde{u}_{i}}{\partial x_{j}}+\frac{\partial \widetilde{u}_{j}}{\partial x_{i}}-\frac{2}{3} \delta_{i j} \frac{\partial \widetilde{u}}{\partial x_{k}}\right)-\frac{2}{3} \delta_{i j} \bar{\rho}_{g} k_{s g s}, \\
q_{i}^{s g s} & =\bar{\rho}_{g}\left(\frac{\nu_{t}}{\operatorname{Pr}_{t}}\right) \frac{\partial \widetilde{h}}{\partial x_{i}}, \\
j_{Y_{\alpha}, i}^{s g s} & =\bar{\rho}_{g}\left(\frac{\nu_{t}}{\mathrm{Sc}_{t}}\right) \frac{\partial \widetilde{Y_{\alpha}}}{\partial x_{i}} .
\end{aligned}
$$

For closure, the turbulent eddy-viscosity is calculated according to

$$
\begin{gathered}
\nu_{t}=\left(C_{s g s} \Delta\right)^{2} \frac{\left(\widetilde{U}_{i j}^{d} \widetilde{U}_{i j}^{d}\right)^{3 / 2}}{\left(\widetilde{S}_{i j} \widetilde{S}_{i j}\right)^{5 / 2}+\left(\widetilde{U}_{i j}^{d} \widetilde{U}_{i j}^{d}\right)^{5 / 4}} \\
4 \text { of } 18
\end{gathered}
$$


with a subgrid-scale model constant $C_{s g s}$ and the filter width $\Delta$. Typically, a value $C_{s g s}=0.520$ is chosen for the WALE constant. However, a value of $C_{s g s}=0.1$ is selected, based on numerical experiments in THETA for simulations involving combustion chambers with highly swirling flows. The turbulent eddy-viscosity is determined by the strain rate tensor

$$
\widetilde{S}_{i j}=\frac{1}{2}\left(\frac{\partial \widetilde{u}_{i}}{\partial x_{j}}+\frac{\partial \widetilde{u}_{j}}{\partial x_{i}}\right)
$$

and the traceless symmetric part of the squared velocity gradient tensor

$$
\widetilde{U}_{i j}^{d}=\frac{1}{2}\left(\widetilde{g}_{i j}^{2}+\widetilde{g}_{j i}^{2}\right)-\frac{1}{3} \delta_{i j} \widetilde{g}_{k k} \quad \text { with } \quad \widetilde{g}_{i j}=\frac{\partial \widetilde{u}_{i}}{\partial x_{j}} \quad \text { and } \quad \widetilde{g}_{i j}^{2}=\widetilde{g}_{i k} \widetilde{g}_{k j} .
$$

\section{B. Turbulent Combustion Modeling}

The FRC model is employed for the calculation of the chemical source terms $\bar{S}_{Y_{\alpha}}^{\text {reac }}$ in the species equations. The conversion of reactants into products is described by a reaction mechanism involving $N_{r}$ elementary reactions and $N_{s p}$ species. In a generalized way, an elementary reaction is given by

$$
\sum_{\alpha=1}^{N_{s p}} \nu_{\alpha, r}^{\prime} \mathcal{R}_{\alpha} \underset{\mathrm{k}_{b, r}}{\stackrel{\mathrm{k}_{f, r}}{\rightleftharpoons}} \sum_{\alpha=1}^{N_{s p}} \nu_{\alpha, r}^{\prime \prime} \mathcal{R}_{\alpha}
$$

with the chemical gas component of a species $\alpha$ indicated by $\mathcal{R}_{\alpha} \cdot{ }^{18}$ The stochiometric coefficients are denoted by $\nu_{\alpha, r}^{\prime}$ for reactants and $\nu_{\alpha, r}^{\prime \prime}$ for products, respectively. The temperature-dependent forward and backward reaction rate constants $k_{f, r}$ and $k_{b, r}$, respectively, are calculated by a modified Arrhenius equation

$$
k_{r}(T)=A_{r} T^{b} e^{-\frac{E_{a, r}}{R_{u} T}}
$$

with the reaction-dependend pre-exponential factor $A_{r}$, temperature exponent $b$, and the activation energy $E_{a, r}$. Both forward and backward reaction rates are related by the thermodynamic equilibrium constant $K_{c, r}=k_{f, r} / k_{b, r}$, which can be determined from the Gibbs free energy 21 The instantaneous, unfiltered species source terms can be computed by

$$
S_{\alpha}^{\mathrm{reac}}=M_{\alpha} \sum_{r=1}^{N_{r}}\left(\nu_{\alpha, r}^{\prime \prime}-\nu_{\alpha, r}^{\prime}\right)\left[k_{f, r} \prod_{\beta=1}^{N_{s p}-1}\left(\frac{\rho_{g} Y_{\alpha}}{M_{\alpha}}\right)^{\nu_{\beta, r}^{\prime}}-k_{b, r} \prod_{\beta=1}^{N_{s p}-1}\left(\frac{\rho_{g} Y_{\alpha}}{M_{\alpha}}\right)^{\nu_{\beta, r}^{\prime \prime}}\right]
$$

To account for the turbulent subgrid-scale fluctuations on the chemical source terms, an APDF approach is employed. Statistical independence of temperature and gas composition is presumed, 22 leading to the filtered species source term

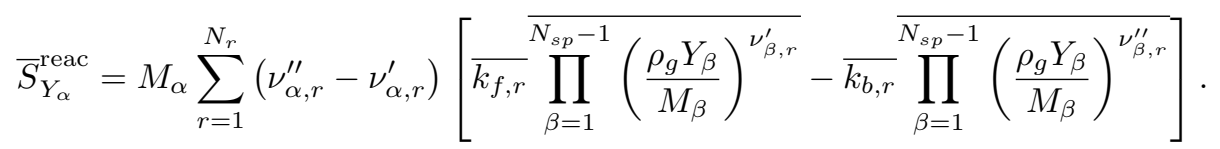

The filtered forward and backward reaction rate constants on the right-hand side of equation (14) are calculated by numerical integration over all realizable states of the sample space variable $\hat{T}$

$$
\overline{k_{r}}=\int_{T_{\min }}^{T_{\max }} k_{r}(\hat{T}) P_{T}(\hat{T}) \mathrm{d} \hat{T},
$$

using a pre-assumed clipped Gaussian Probability Density Function (PDF) for the temperature

$$
P_{T}(\hat{T})=\frac{1}{\sqrt{2 \pi \sigma_{T}}} \exp \left[-\frac{(\hat{T}-\tilde{T})^{2}}{2 \sigma_{T}}\right]
$$


For further information concerning the clipping of $P_{T}(\hat{T})$ on the interval $\left[T_{\min }, T_{\max }\right]$ we refer to the work of Gerlinger et al 223 The clipped Gaussian PDF is determined by its first moment $\tilde{T}$ and its second moment $\sigma_{T}$, the temperature variance. The latter is estimated by the solution of the following transport equation $23 \sqrt{24}$

$$
\frac{\partial}{\partial t}\left(\bar{\rho} \sigma_{T}\right)+\frac{\partial}{\partial x_{i}}\left(\bar{\rho}_{g} \tilde{u}_{i} \sigma_{T}\right)=\frac{\partial}{\partial x_{i}}\left(\bar{\rho}_{g} \frac{\nu_{t}}{\operatorname{Pr}_{T}} \frac{\partial \sigma_{T}}{\partial x_{i}}\right)+2 \bar{\rho}_{g} \frac{\nu_{t}}{\operatorname{Pr}_{T}}\left(\frac{\partial \tilde{T}}{\partial x_{i}}\right)^{2}-C_{T} \bar{\rho}_{g} \frac{\sigma_{T}}{\tau_{T}}
$$

with the turbulent timescale $\tau_{T}$ and the model constant $\left.C_{T}=1\right]$ The filtered species production terms $\prod_{\beta=1}^{N_{s p}-1}\left(\frac{\rho_{g} Y_{\beta}}{M_{\beta}}\right)^{\nu_{\beta, r}^{\prime}}$ are calculated similarly through the utilization of a multivariate $\beta$-PDF for the species mass fractions, which goes back to the work of Girima: 25

$$
P_{Y}(\hat{\mathbf{Y}})=\frac{\Gamma\left(\sum_{\alpha=1}^{N_{s p}} \beta_{\alpha}\right)}{\prod_{\alpha=1}^{N_{s p}} \Gamma\left(\beta_{\alpha}\right)}\left[\delta\left(1-\sum_{\alpha=1}^{N_{s p}} \hat{Y}_{\alpha}\right) \prod_{\alpha=1}^{N_{s p}} \hat{Y}_{\alpha}^{\beta_{\alpha}-1}\right]
$$

with

$$
\beta_{\alpha}=\tilde{Y}_{\alpha}\left(\frac{\sum_{\gamma=1}^{N_{s p}} \tilde{Y}_{\gamma}\left(1-\tilde{Y}_{\gamma}\right)}{\sigma_{Y}}-1\right)
$$

The major advantage of this approach is, that this distribution is fully parametrized by the first species moments $\tilde{Y}_{\alpha}$ and the sum of species mass fraction variances $\sigma_{Y}$, which is determined from the folllowing transport equation 24

$$
\frac{\partial}{\partial t}\left(\bar{\rho} \sigma_{Y}\right)+\frac{\partial}{\partial x_{i}}\left(\bar{\rho}_{g} \tilde{u}_{i} \sigma_{Y}\right)=\frac{\partial}{\partial x_{i}}\left(\bar{\rho}_{g} \frac{\nu_{t}}{\operatorname{Pr}_{T}} \frac{\partial \sigma_{Y}}{\partial x_{i}}\right)+2 \bar{\rho}_{g} \frac{\nu_{t}}{\operatorname{Pr}_{T}} \sum_{\alpha=1}^{N_{s p}}\left(\frac{\partial \tilde{Y}_{\alpha}}{\partial x_{i}}\right)^{2}-C_{Y} \bar{\rho}_{g} \frac{\sigma_{Y}}{\tau_{T}}
$$

A value of 2.0 has been chosen for the model constant $C_{Y}$ [18 For further details regarding the APDF model we refer to the literature.22 26 A stiff-chemistry solver is used to solve the reduced chemical mechanism for Jet-A1 combustion with 59 species and 372 elementary reactions from Slavinskaya et al.27 29] This mechansim has already been validated extensively by various authors. 29 The vaporized jet fuel is represented by four surrogate species, namely $\mathrm{N}$-dodecane $\mathrm{nC}_{12} \mathrm{H}_{26}$, iso-octane $\mathrm{C}_{8} \mathrm{H}_{18}$, cyclo-hexane $\mathrm{C}_{6} \mathrm{H}_{12}$ and toluene $\mathrm{C}_{7} \mathrm{H}_{8}$. These represent the most important chemical classes n-alkanes, iso-alkanes, cyclo-alkanes and aromatics, respectively. 33

\section{Dispersed Phase Modeling}

To compute the dispersed liquid phase of the fuel, the DLR in-house code SPRAYSIM is employed, which is based on a Lagrangian particle tracking method. Droplets are approximated through mathematical parcels, which are transported by the flow field and provide point sources and point forces to the gas field. For every parcel $p$, the following coupled ordinary differential equations are solved for its position $\mathbf{x}_{p}$, velocity $\mathbf{u}_{p}$ and diameter $d_{p}^{33}$

$$
\begin{aligned}
\frac{\mathrm{d} \mathbf{x}_{p}}{\mathrm{~d} t} & =\mathbf{u}_{p} \\
\frac{\mathrm{d} \mathbf{u}_{p}}{\mathrm{~d} t} & =\frac{3}{4} \frac{c_{d}}{d_{p}} \frac{\rho_{g}}{\rho_{l}}\left|\mathbf{u}_{g}-\mathbf{u}_{p}\right| \cdot\left(\mathbf{u}_{g}-\mathbf{u}_{p}\right)+\left(1-\frac{\rho_{g}}{\rho_{l}}\right) \mathbf{g}, \\
\frac{\mathrm{d} d_{p}}{\mathrm{~d} t} & =-\frac{1}{3} \frac{d_{p}}{\rho_{l}} \frac{\mathrm{d} \rho_{l}}{\mathrm{~d} t}-\frac{2}{\rho_{l}} \frac{\dot{m}_{v a p}}{\pi d_{p}^{2}}
\end{aligned}
$$

The vector $\mathbf{g}$ represents the gravitational force. The indices $l$ and $g$ denote the liquid and the gaseous phase, $c_{d}$ is the particle drag coefficient and $\dot{m}_{v a p}$ the vapor mass flow rate from the surface particle in case of evaporation. To account for the influence of unresolved turbulent fluctuations in the subgrid-scale on the droplet dispersion, a variant of the stochastic dispersion model of Bini and Jones 36 is employed, for which an additional stochastic droplet acceleration term is added to equation 21b. The Cascade Atomization and Droplet Breakup (CAB) model of Tanner ${ }^{\sqrt[37]{3}}$ is utilized for modeling droplet breakup. The vaporiazion 
model of Eckel et al $\sqrt[33]{34}$ is used to account for droplet vaporization. It combines the uniform temperature model of Abramzon and Sirignand ${ }^{38}$ and the Continuous Thermodynamics Model (CTM) of Doué $\sqrt[39]{ }$ Jet-A1 fuel components, which have been determined experimentally by GCxGC chromatography, 40 are grouped into four chemical families according to their molecular structures (n-alkanes, iso-alkanes, cyclo-alkanes and aromatics). Each chemical family $j$ is represented by means of a $\Gamma$-PDF with the molar weight as distribution parameter. These continuous distribution functions are employed for the calculation of phyical properties of the fuel, such as the specific heat of evaporation $\Delta h_{e v a p, j}$ and the specific isobaric heat capacities $c_{p, j}$. In order to couple the fuel vapor phase to the gas field, the four chemical classes are mapped to a fuel surrogate species, respectively. The change in fuel composition $Y_{j, l}$ and droplet temperature $T_{p}$ is calculated by

$$
\begin{aligned}
\frac{\mathrm{d} Y_{j, l}}{\mathrm{~d} t} & =\frac{6 \dot{m}_{v a p}}{\rho_{l} \pi d_{p}^{3}}\left(Y_{j, l}+\frac{Y_{j, l}^{\infty}-Y_{j, l}^{S}\left(1+B_{M}\right)}{B_{M}}\right) \\
\frac{\mathrm{d} T_{p}}{\mathrm{~d} t} & =-\frac{6}{c_{p_{l}}} \frac{\dot{m}_{v a p}}{\rho_{l} \pi d_{p}^{3}} \sum_{j=1}^{N_{s p}}\left(\Delta h_{v a p, j}-\frac{c_{p, j}\left(T_{g}^{\infty}-T_{g}^{S}\right)}{B_{T}}\right),
\end{aligned}
$$

with the mass fractions $Y_{j, l}^{\infty}$ in the gas field and the mass fraction $Y_{j, l}^{S}$ on the droplet surface. The component PDFs are determined locally by solving ordinary differential equations for their first and second moments. For further details on the calculation of the PDF-moments, as well as the calculation of the vaporizing mass flow rate $\dot{m}_{v a p}$, the specific heat of evaporation $\Delta h_{e v a p, j}$ and the specific isobaric heat capacities $c_{p, j}$, we refer to the works of Eckel et al ${ }^{33}$ They also describe the calculation of the particle source-terms $\bar{S}_{i}^{d}$, that are introduced in Equation (1).

\section{Results for the Non-Reactive Operating Point}

\section{A. Non-Reactive Setup and Boundary Conditions}

For the non-reactive operating point of the burner, three different meshes are examined, which differ mainly in the element-resolution in the flame-zone of the combustion chamber, as shown in Fig. 2 and Tab. 2 . Sufficiently low $y^{+}$values close to wall boundaries are obtained due to the usage of suitable prismatic elements. The air mass flow rate is prescribed at the inlet of the plenum and adiabatic boundary conditions are applied to the walls. Concerning the averaging process of the flow field quantities, a flow through time of $\tau_{f}=0.04 \mathrm{~s}$ is chosen, based on the work of Andreini et al. ${ }^{7}$ Two average flow through times are used for the initialization of the flow and additional three flow through times for the following averaging process of mean flow quantities and respective Root-Mean-Squared (RMS) values. For each mesh, a timestep of $10^{-6} \mathrm{~s}$ is employed, leading to 200,000 timesteps per calculation. The simulations were performed on 12 Intel Ivy-Bridge E5 nodes each with 12 cores, $2.4 \mathrm{GHz}$ and $64 \mathrm{~GB}$ memory. Roughly 96, $000 \mathrm{CPUh}$ were required for one single simulation.

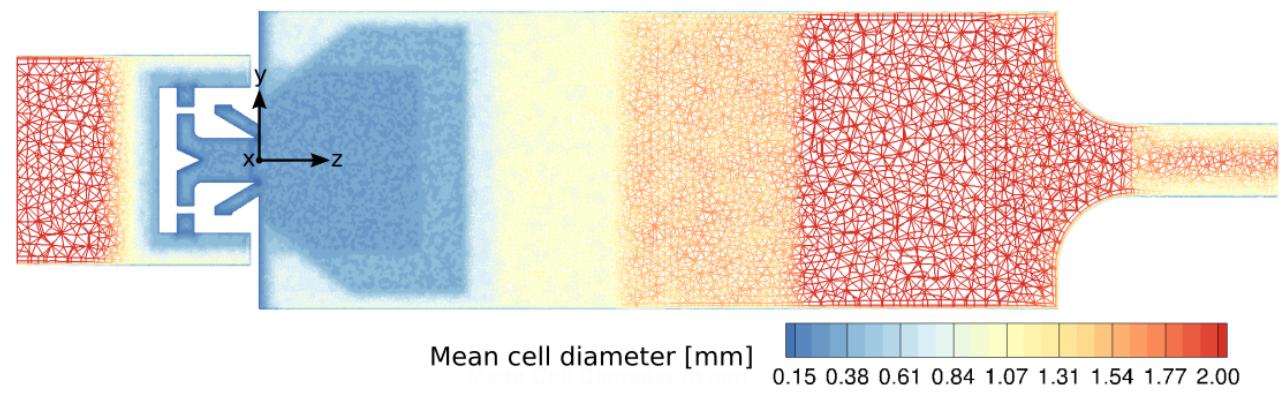

Figure 2: Mean cell diameters for the coarse mesh with 3.9 million nodes. 
Table 2: Mesh features for the Generic Single Sector Combustor model.

\begin{tabular}{lccc}
\hline & Coarse & Medium & Fine \\
\hline Number of nodes & 3.9 million & 5.5 million & 7.3 million \\
Number of cells & 17.3 million & 24.2 million & 34.3 million \\
Prism layers & 6 & 8 & 8 \\
Smallest element mean diameter & $0.7 \mathrm{~mm}$ & $0.5 \mathrm{~mm}$ & $0.4 \mathrm{~mm}$ \\
\hline
\end{tabular}

\section{B. Non-Reactive Flow Field}

The highly turbulent flowfield in the combustion chamber is shown in Figs. 3a |3c through the instantaneous axial velocity field. The co-rotating swirler applies tangential momentum to the air fed by the plenum, which generates a radial pressure gradient. Downstream from the nozzle exit, the tangential velocity is reduced due to the gas-expansion, which causes a decay of the radial distribution of the pressure gradient and, thus, a negative axial pressure gradient in the vicinity of the burner axis. This induces a reverse flow and the formation of the central recirculation zone $\frac{41}{11}$ which penetrates deep into the air injection nozzle. The burners confined geometry forces the entering air flow to a sudden expansion and thus leads to the formation of corner recirculation zones ${ }^{[42}$ Strong shear layers develop due to the velocity difference between the entering air jet and both the corner and center recirculation zones. $\stackrel{43}{[3}$ Vortex shedding occurs at the combustion chamber

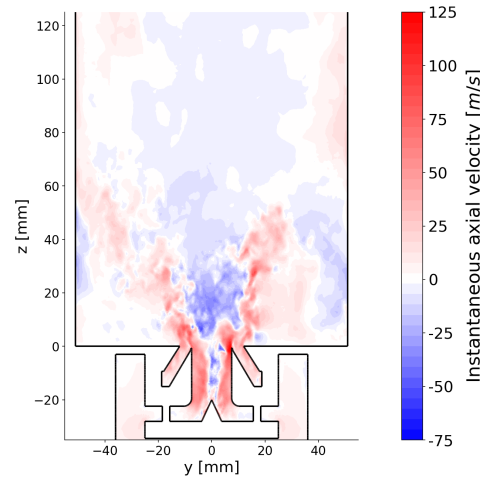

(a) 3.9 million nodes

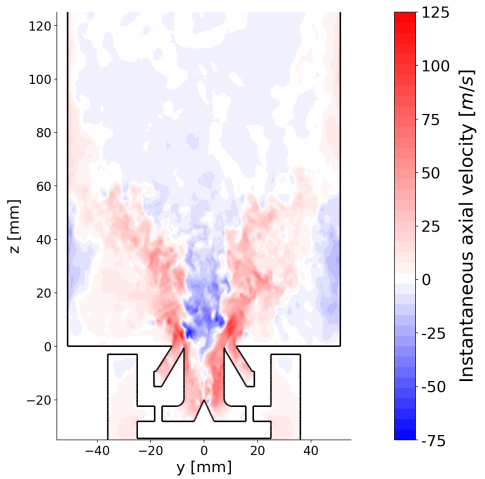

(b) 5.5 million nodes

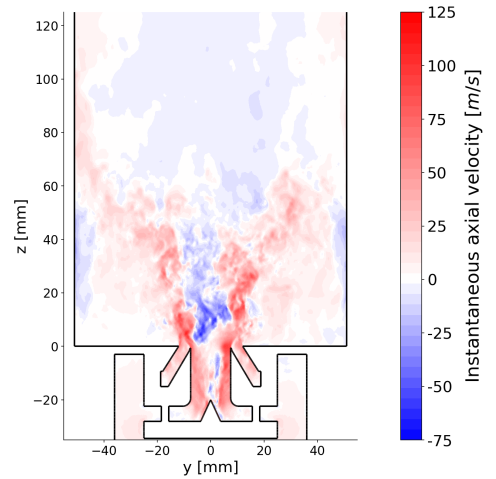

(c) 7.3 million nodes

Figure 3: Instantaneous axial velocity.

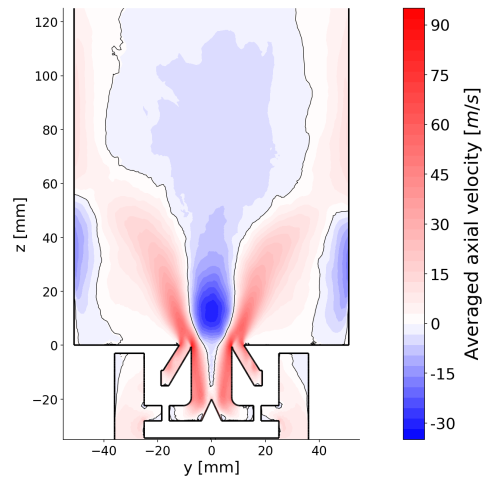

(a) 3.9 million nodes

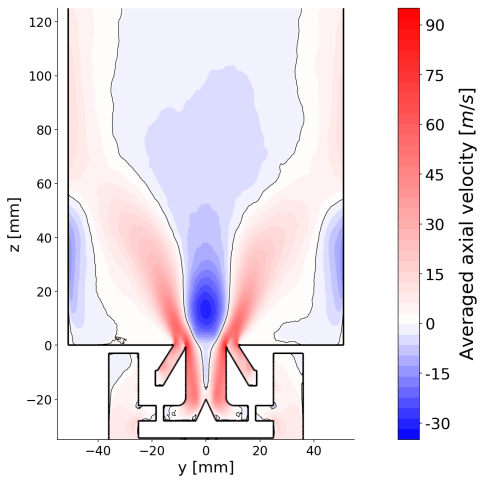

(b) 5.5 million nodes

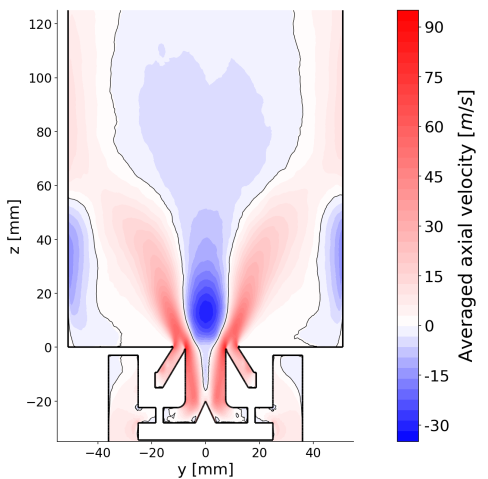

(c) 7.3 million nodes

Figure 4: Averaged axial velocity. Black lines indicate transition from positive to negative values. 


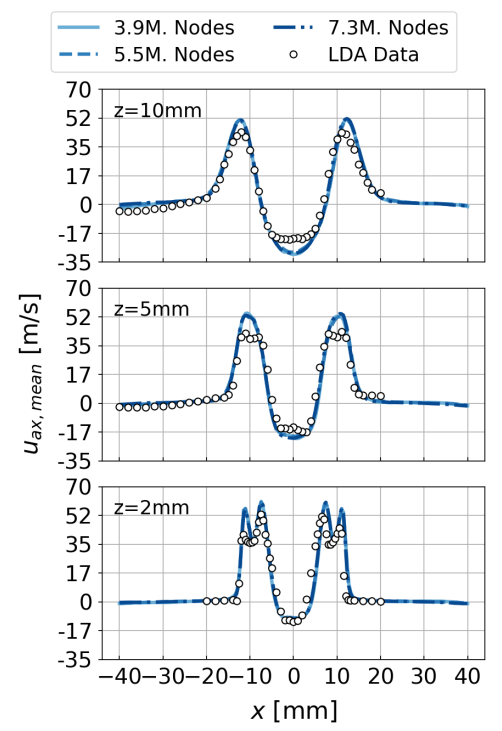

(a) Axial

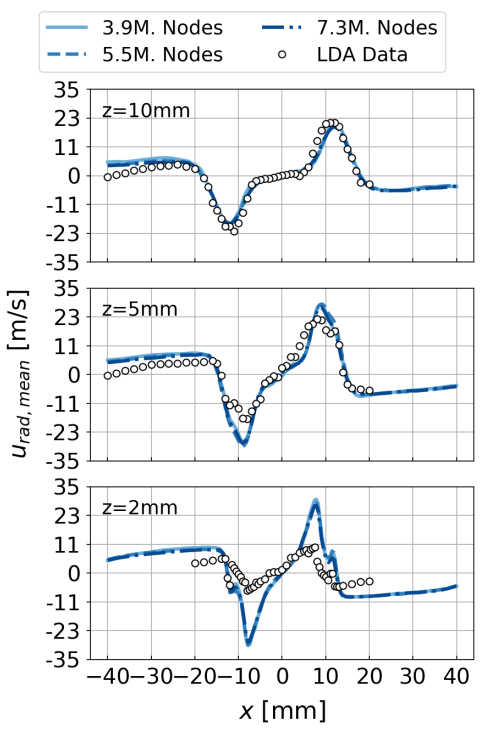

(b) Radial

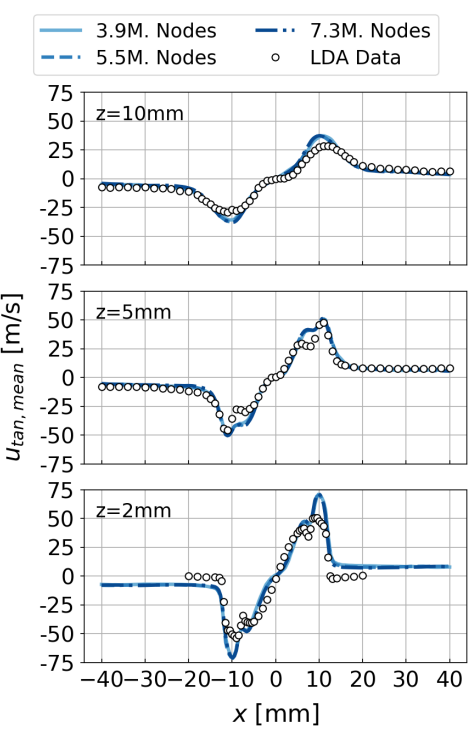

(c) Tangential

Figure 5: Mean velocity profiles at various heights obtained from LES computations, and with LDA measurements from Meier et al! 10

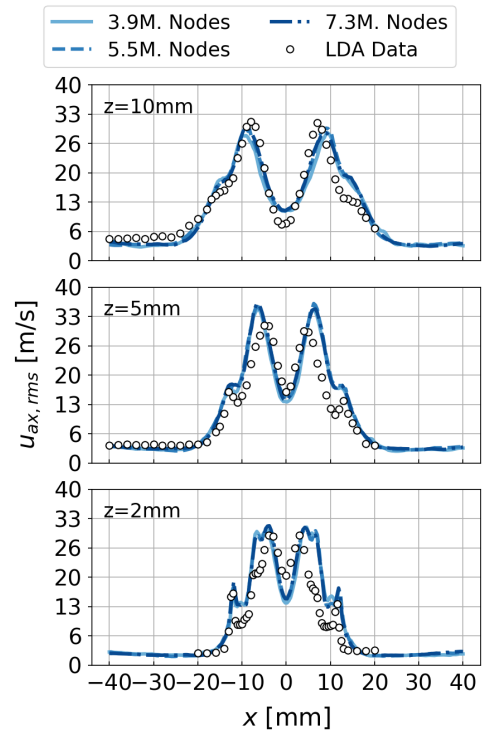

(a) Axial

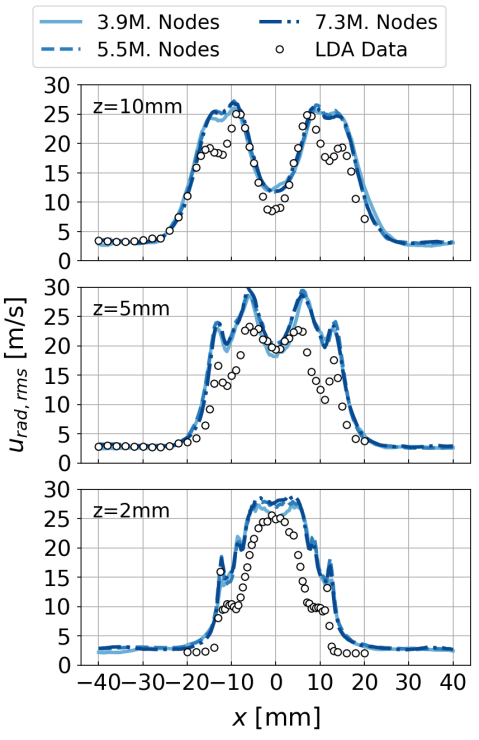

(b) Radial

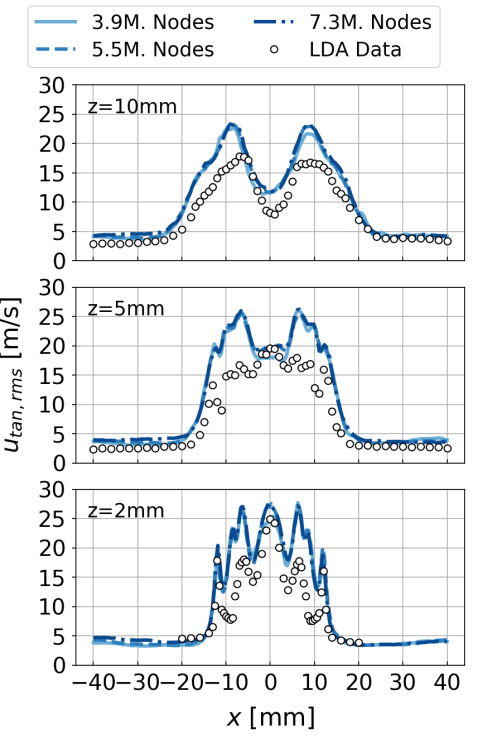

(c) Tangential

Figure 6: RMS velocity profiles at various heights obtained from LES computations, and with LDA measurements from Meier et al 10

inlet due to the sharp nozzle edges. This leads to the generation of eddies which decay into smaller structures through the interaction with the shear layers. The overall flow field is given in Figs. 4a $4 \mathrm{~b}$ for all meshes in the form of averaged axial velocities. All meshes predict similar flow features, such as minimum and maximum averaged axial velocities of $-30 \mathrm{~m} / \mathrm{s}$ and $90 \mathrm{~m} / \mathrm{s}$, respectively. A strong central vortex in the injector causes the deep penetration of the central recirculation zone into the nozzle with a stagnation point at $z \approx-15 \mathrm{~mm}$. For all simulations, this central vortex core performs a precession around the burner axis. The results of the non-reacting test case are compared to experimental LDA velocity profiles.10 ${ }^{[0}$ Mean and 
RMS profiles of axial, radial and tangential velocity are shown at various heights $z$ in Figs. 5 and 6 . The position $z=0 \mathrm{~mm}$ refers to the entrance plane of the combustion chamber. The calculated mean values of all velocity components agree excellently well with the experimental data for the results from all three meshes. The opening angle is captured by all simulations, as well as minimum and maximum velocity values. Only very close to the nozzle exit, at $z=2 \mathrm{~mm}$, the radial and tangential profiles are slightly overpredicted. Similarly good results are obtained for the RMS profiles, whereas the best agreement is obtained for the axial velocity profiles. The radial and tangential RMS velocity profiles are slightly overestimated. Nevertheless, the overall agreement with the experiments is very good.

\section{Results for the Reactive Operating Point}

\section{A. Boundary Conditions}

Since reasonable results could be obtained for the non-reacting operating point with all three grids, the coarse grid with 3.9 million nodes is used for the simulation of the reactive operating point, in order to reduce computation times. Similarly to the work of Jones et al., 6 a droplet temperature of $295 \mathrm{~K}$ and droplet velocities of $50 \mathrm{~m} / \mathrm{s}$ are prescribed as initial condition, with an injection angle of $160^{\circ}$. Spray droplets are injected into the combustion chamber at random positions on an annular surface $1 \mathrm{~mm}$ downstream of the fuel injector prefilmer lip. A Rosin-Rammler PDF is utilized to generate the initial droplet size distribution with a characteristic diameter $D_{v 50}=17.6 \mathrm{~mm}$ and a form factor $q=3.9$. These values have been obtained from parametric studies on a coarser grid of the burner. Isothermal wall boundary conditions are employed, with temperatures of $550 \mathrm{~K}$ upstream of the combustion chamber, $1000 \mathrm{~K}$ in the vicinity of the flame zone and $800 \mathrm{~K}$ at the combustor walls downstream of the flame zone. Since this operating point features higher velocities than the non-reactive case, a reduced flow through time $\tau_{f} \approx 0.01 \mathrm{~s}$ is used for the temporal averaging of the simulation data and a timestep of $3 \times 10^{-7} \mathrm{~s}$ is chosen. The flow field of the non-reactive operating point is used as an initial solution for the reactive simulation, so that it is sufficient to compute only one flow through time for the initialization process. The averaging of temporal mean and RMS variables is performed for another two flow through times, due to the high computational effort required for the employed FRC approach. This leads to a total amount of 100,000 timesteps to compute. In contrast to the non-reactive operating point, the calculation time for a single iteration increases by a factor of five. This is because of the additional 60 transport equations, which have to be solved in the case of FRC and APDF closure. The same computing capacities as for the non-reactive operating point were used, leading to a total computation time of approximately 280,000 CPUh.

\section{B. Reactive Flow Field}

Snapshots of the instantaneous flowfield for the reactive operating point are shown in Fig. 7, while averaged flow quantities are given in Fig. 8. As the droplets are injected into the combustion chamber, they start to disperse through the interaction with turbulent flow structures. The droplets heat up due to surrounding hot exhaust gases, as given in Fig. $7 \mathrm{C}$, and finally evaporate. Vaporized fuel further mixes with the gaseous flow and reacts with the oxygen of the incoming air. The reaction zone is shown in Fig. 8c by means of averaged $\mathrm{OH}$ mass fractions. The reactive flow field is characterized by similar flow features as before in the non-reactive case. However, the central recirculation zone impinges less far into the nozzle of the air injector. High axial velocities are present at the combustor inlet due to strong blockage effects from the central recirculation zone. The blockage is increased in the case of combustion because of the expansion of hot exhaust gases. This also causes a broader opening angle of the entering air jet. Both central and corner recirculation zones act as major contributors to the flame stabilization process because of the transport of hot exhaust gases into the oxidizer stream, which favors the evaporation of injected droplets. The interaction of shedded vortex structures from the inlet edges with both recirculation zones in the vicinity of the shear layers leads to an enhanced turbulent mixing process and a further stabilization of the lifted flame. The wrinkled flame front develops downstream of $z \approx 20 \mathrm{~mm}$ as a results of the enhanced turbulent transport and because of the presence of vaporized fuel. Pockets of hot temperatures are convected further downstream and break up due to the turbulent decay of vortical structures. The averaged axial velocity field in Fig. 8a indicates, that the flow field is fully converged. However, the averaged temperature field shows a slightly non-symmetric shape, indicating that a fully statistically converged solution is not yet reached. 


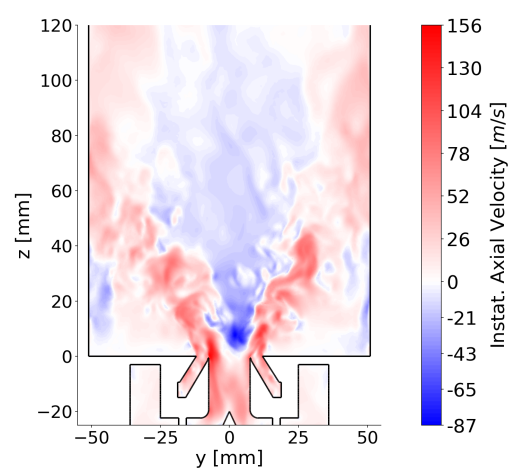

(a) Axial gas velocity

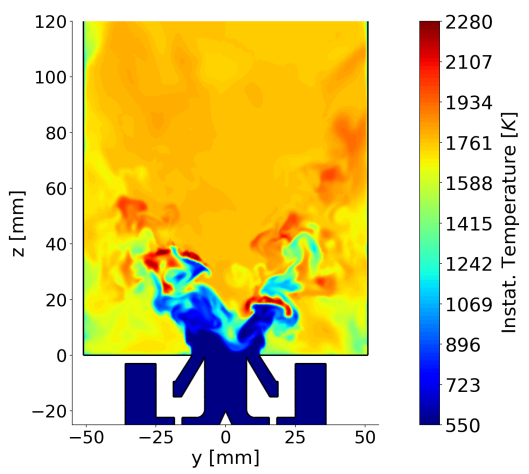

(b) Gas temperature

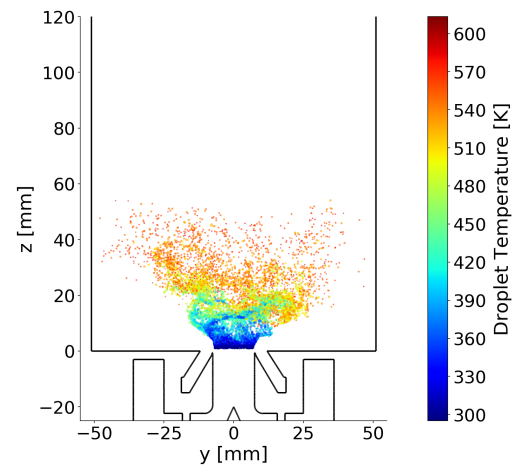

(c) Droplet temperature

Figure 7: Snapshop of the instantaneous flow field for the reactive operating point.

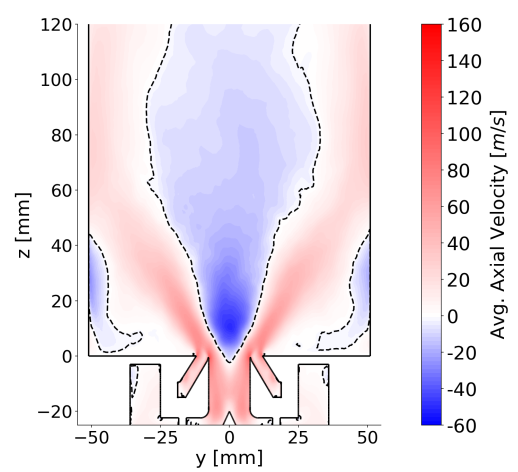

(a) Axial gas velocity

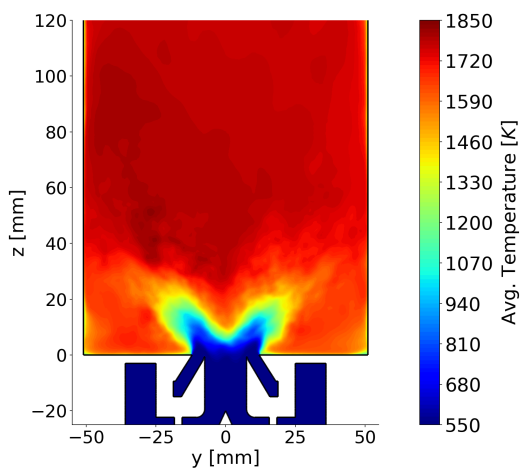

(b) Gas temperature

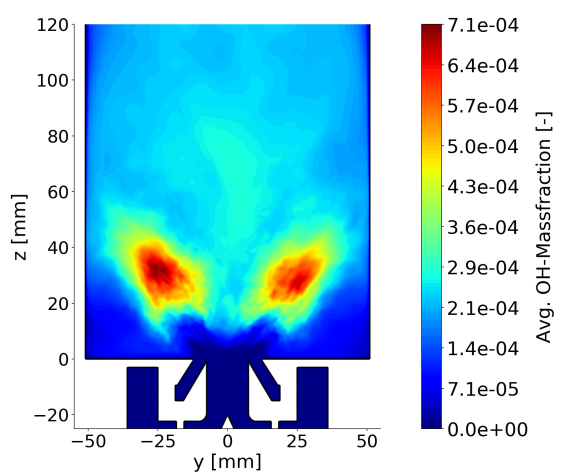

(c) $\mathrm{OH}$ mass fractions

Figure 8: Averaged flow field for the reactive operating point.

\section{Spray Evaporation Process}

The spray evaporation process is investigated in Fig. 9. where fuel properties have been extracted from 10, 000 representative particles of a single LES timestep. Liquid fuel mass fractions for each chemical family of the CTM model are shown, as well as droplet diameters. Every point corresponds to a particle, whereas each property is plotted against individual droplet temperatures. The particle compostions remain constant up to droplet temperatures of approximately $400 \mathrm{~K}$, while particle diameters persist up to approximate $525 \mathrm{~K}$. The change of the various liquid mass fractions is related to the different evaporation rates of the employed chemical families and the corresponding differences in the vapor pressure. Cyclo-alkanes evaporate first, since this family holds molecules with the shortest chain lengths, which results in high vapor pressures ${ }^{34}$ The n-alkane family and aromatics vaporize at higher temperatures, due to longer chain-lengths and different molecular structures, respectively ${ }^{34}$ The iso-alkanes evaporize last, which is expressed by the increase in the liquid fuel mass fraction at temperatures above $550 \mathrm{~K}$. This is due to the fact, that remaining chemical families are already evaporated to a certain extent.

Fig. 10 shows droplet velocities and SMDs at five different axial locations in the combustion chamber. Quadrilateral registration planes with a size of $20 \times 20 \mathrm{~mm}^{2}$ are defined at the axial positions of the PDA measurements and are discretized using a grid of $225 \times 225$ hexahedral elements. Every time a parcel passes a plane, its properties are assigned to the corresponding element. Ensemble-averages of the droplet properties, shown as blue dots at respective radial coordinates, are calculated from all parcels that have passed during two flow through times. The blue lines represent mean-values over the radial coordinate, calculated from the scattered point data. Black dots denote PDA measurements $10[11$ A good agreement between calculated and measured SMD profiles is obtained at all heights. A low amount of particles is sampled near the burner 


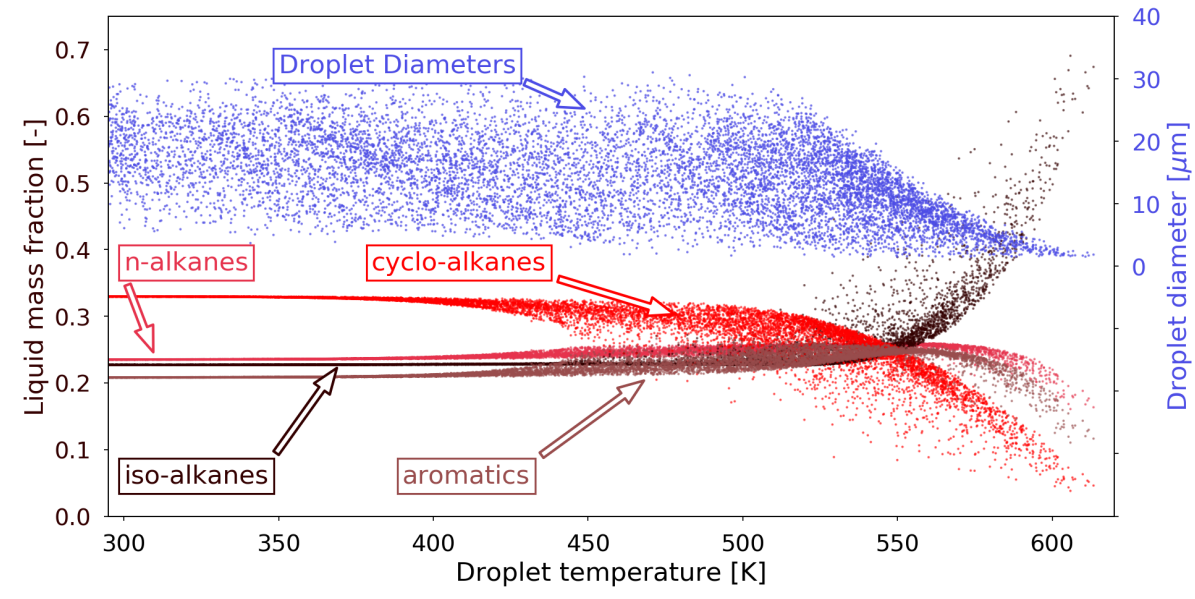

Figure 9: Instantaneous particle diameters and liquid mass fractions of the respective chemical families over droplet temperature. The increase of iso-alkane liquid mass fractions above $525 \mathrm{~K}$ is due to other liquid components being already evaporated.

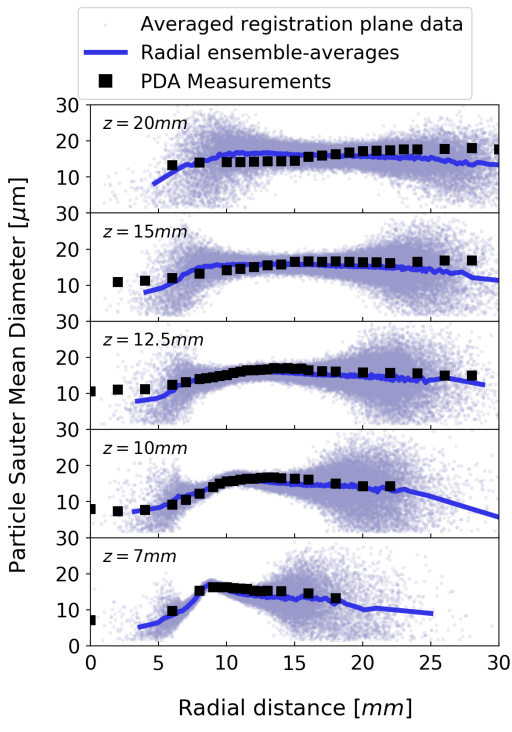

(a) Sauter mean diameters



(b) Axial velocities

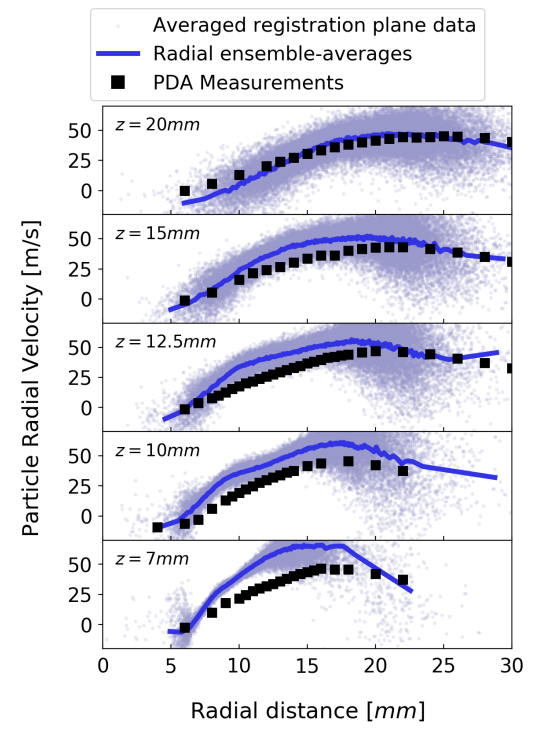

(c) Radial velocities

Figure 10: Averaged droplet profiles at various axial positions, compared to data from PDA experiments 10,11 Blue dots represent averaged values at positions in registration planes used for the sampling of droplet properties. Blue lines denote radial ensemble-averages of blue dots.

axis at radial coordinates $r<5 \mathrm{~mm}$. This is related to the low averaging time of the simulation compared to the sampling time scales in the measurements. The shape and opening angles of the velocity profiles agree well with the experimental results. The profiles also imply an accurate prediction of the gaseous velocity field, which underlies the particle trajectories. However, axial and radial velocities are slightly overpredicted in the vicinity of the inlet. This has also been observed for the non-reactive operating point.

Normalized averaged liquid volume fractions from LES and normalized planar Mie scattering data from experiments $10[1]$ are compared in Fig. 11. Planar Mie scattering is utilized for the imaging of the liquid fuel phase. Even though the comparison between both datasets is only qualitative, the results show a good agreement in terms of opening angle and length of the V-shaped spray cone. Similar to Fig. 10a, almost no droplets are present on the centerline in the simulation. The red line, which refers to a temperature of 


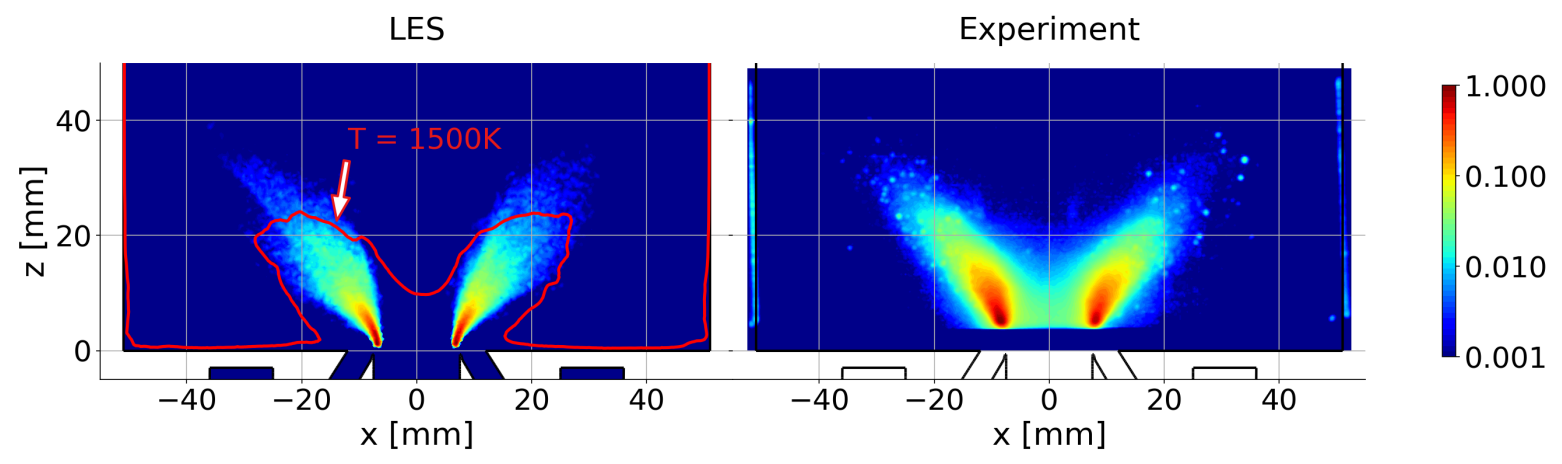

Figure 11: Normalized liquid volume fraction from LES compared to normalized planar Mie scattering from experiments 10$] 11$ The red line indicates averaged temperatures of $1500 \mathrm{~K}$.

$1500 \mathrm{~K}$, indicates that droplets are still present at high temperatures. This has also been noticed in LES results of Jones et al ${ }^{6}$

\section{Reaction Zone and Temperature Field}

The reaction zone of the burner is illustrated by the averaged heat release from LES in Fig. 12 and compared to experimental $\mathrm{OH}$ chemiluminescence measurements 10 [11 Both simulation and experimental values are normalized to their maximum levels. The averaged flame structure is characterized by a V-shaped, lifted reaction zone, whose overall shape and opening angle is captured well by the LES. Also, the lift-off height of the flame is predicted with a reasonable accuracy, where the anchoring position of the lifted flame is located at a height of $z \approx 15 \mathrm{~mm}$ in the simulation, compared to $z \approx 20 \mathrm{~mm}$ in the experiment.

The time averaged temperature field from the LES is assessed in Fig. 13, alongside the experimental temperature field, which has been obtained from OH-PLIF measurements 10 11 The experimental data is clipped at a lower bound of $1500 \mathrm{~K}$, because of the rapid decrease in $\mathrm{OH}$ concentrations with temperatures in the experiments 10 The temperature field shows major discrepancies between experiment and simulation. The overall temperature levels seem to be underestimated, especially in the vicinity of the temperature lobes, which occur downstream of the lifted reaction zone at $z \approx 35 \mathrm{~mm}$. LES temperatures near the centerline are somewhat higher than in the experiments. The temperature lobes in the LES show a non-symmetric shape due to the fact that the simulation is not yet statistically fully converged. However, the experimental temperature distribution is associated with some degree of uncertainty, because it was calculated from $\mathrm{OH}$ concentrations, which have been determined from simultaneous PLIF and absorption measurements. According to Meier et al. $\frac{10}{10}$ inferring temperatures from $\mathrm{OH}$ concentrations in fuel rich regions can lead to overestimations of temperatures of about 100K. Since the droplet profiles in Fig. 10 indicate a correct distribution of the liquid phase, a cause for the large temperature disparities could be the applied boundary conditions in the LES, especially the wall temperatures. The experimental design contains cooling air ducts which direct film cooling air from the plenum to the combustion chamber walls (see Fig. 1). These ducts have not been modeled in the LES in order to reduce the computatinal effort required. The influence of the film cooling air is further investigated in Fig. 14 by means of averaged and RMS profiles of velocitites and temperatures at various axial heights in the combustion chamber. The profiles are compared to LES results from Jones et al $[\sqrt{6}$ and averaged experimental temperature profiles, which are obtained from the OH-PLIF contours in Fig. 13. The film cooling air was modeled in the reference work using additional inlet boundary conditions with presumed mass fluxes. Concerning the averaged axial velocities, the present LES offers a broader opening angle of the entering air jet than the reference LES. The RMS velocities agree well in terms of distribution and absolute values for both simulations. Similar averaged temperature levels are obtained on the centerline of the burner for $z \geq 20 \mathrm{~mm}$ and a good agreement is obtained between the different RMS temperature profiles. There are larger discrepancies between both LES close to the walls, probably because of cooling air streams. However, it seems that the influence of the cooling ducts on the main flow is rather low, since our averaged temperature profiles show less discrepancies near the walls with respect to the experiment. 


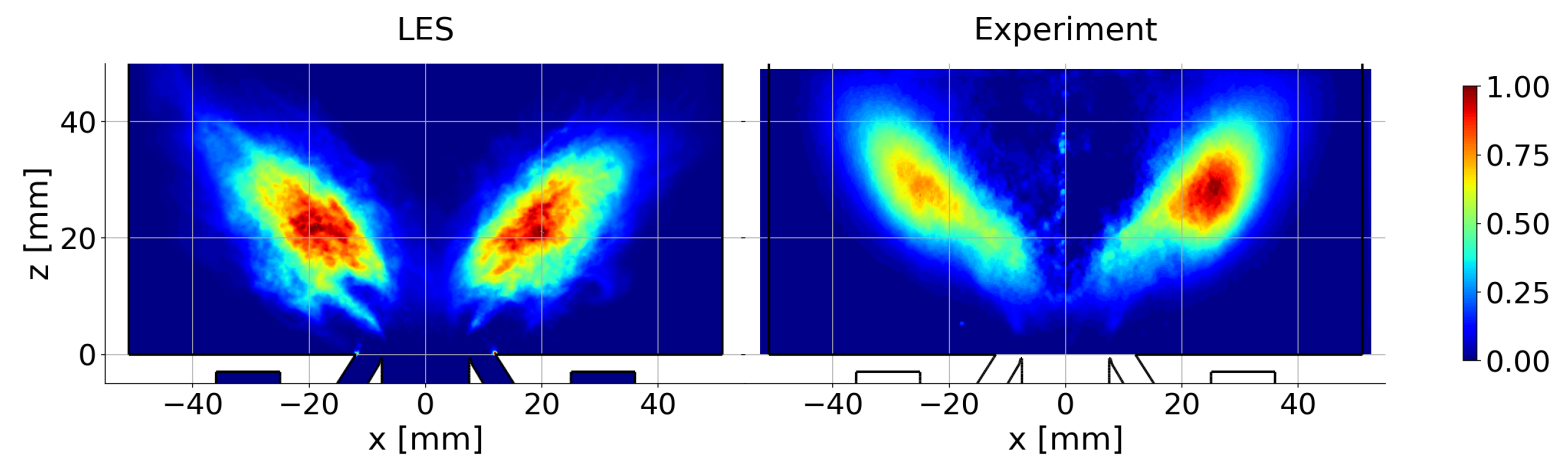

Figure 12: Normalized averaged heat release from LES compared to normalized $\mathrm{OH}$ chemiluminescence from experiments $10[11$

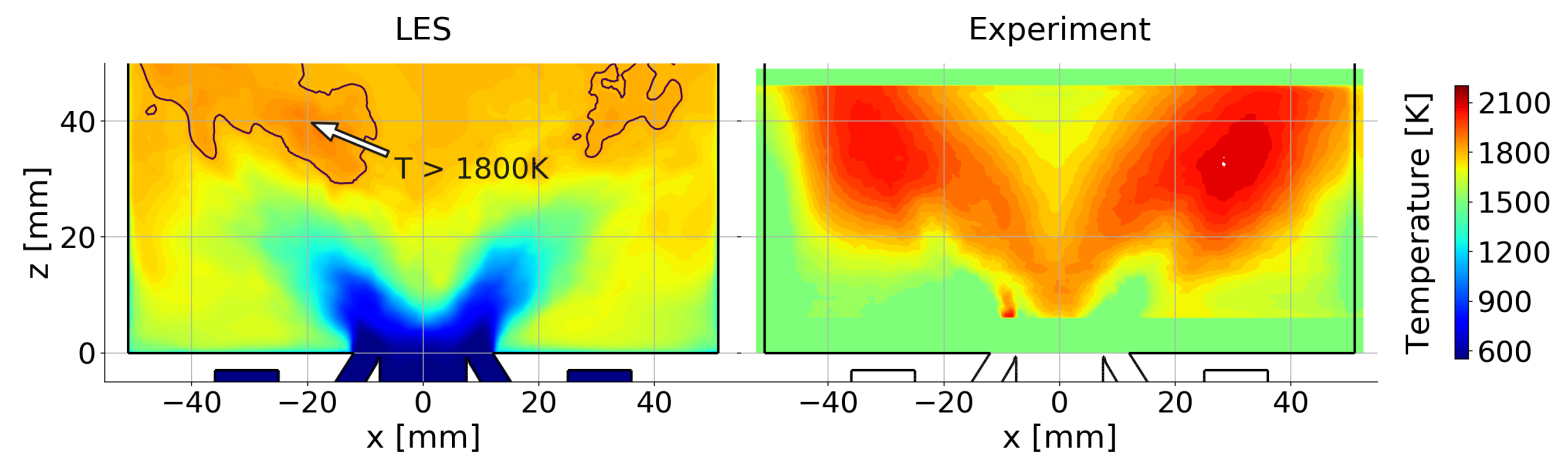

Figure 13: Averaged temperature field from LES compared to the temperature field from OH-PLIF measurements $10[11$

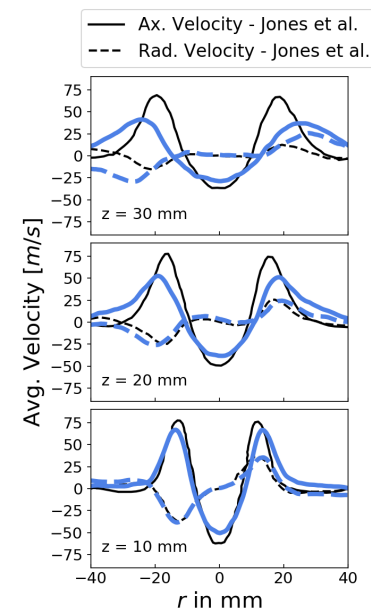

(a) Velocity profiles

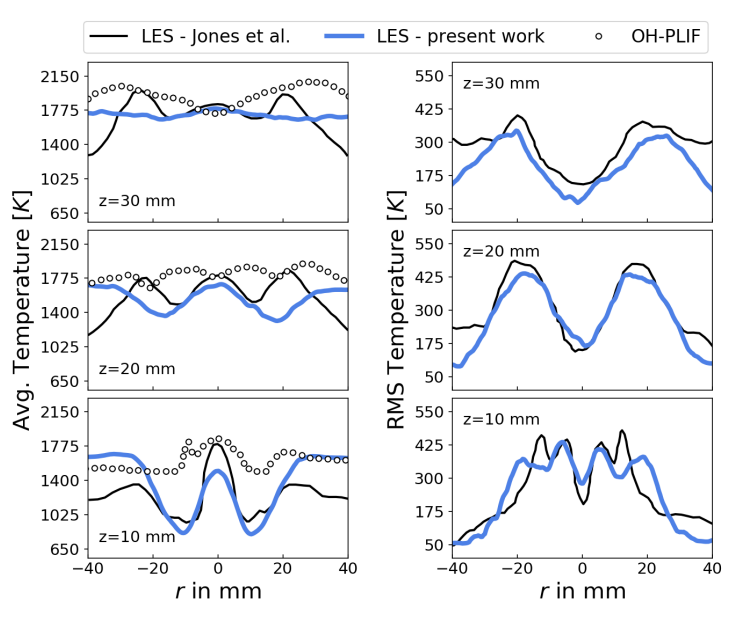

(b) Temperature profiles

Figure 14: Profiles of axial velocity and temperature at different axial locations in the combustion chamber. 


\section{E. Mixing Process and Flame Properties}

The mixing process in the combustion chamber is further analyzed with the mixture fraction definition of Bilger et al. $[4]$

$$
\xi=\frac{\frac{2 Y_{\mathrm{C}}}{M_{\mathrm{C}}}+\frac{Y_{\mathrm{H}}}{2 M_{\mathrm{H}}}-\frac{2\left(Y_{\mathrm{O}}-Y_{\mathrm{O}, \mathrm{Ox}}\right)}{M_{\mathrm{O}}}}{\frac{2 Y_{\mathrm{C}, \mathrm{Fuel}}}{M_{\mathrm{C}}}+\frac{Y_{\mathrm{H}, \mathrm{Fuel}}}{2 M_{\mathrm{H}}}+\frac{2 Y_{\mathrm{O}, \mathrm{Ox}}}{M_{\mathrm{O}}}},
$$

which is based on the element mass fractions of carbon $Y_{\mathrm{C}}$, hydrogen $Y_{\mathrm{H}}$ and oxygen $Y_{\mathrm{O}}$. The definition yields a value of $\xi=1$ in the fuel and $\xi=0$ in the oxidizer. The subscripts Fuel and $O x$ refer to conditions on the fuel and oxidizer side, respectively. Initial liquid fuel mass fractions of $23.51 \%$ n-alkanes, $22.70 \%$ isoalkanes, $32.97 \%$ cyclo-alkanes and $20.82 \%$ aromatics are utilized for the fuel side. A stoichiometric mixture fraction of $\xi_{s t}=0.083$ is obtained for the employed pressure of four bar. The spatial distribution of the instantaneous mixture fraction in the combustion chamber is illustrated in terms of colored lines in Fig. 15a. The instantaneous temperature field is highlighted in grey contours.

Different regions in the combustion chamber, namely the central mixing zone, the external recirculation zones, and the upper mixing zone, are denoted by colored rectangles. The thermo-chemical state space of these areas is shown in Fig. 15c. Every point corresponds to a computational cell in the $3 D$ space of the simulation. The colors indicate the respective combustor region, whereas the points in the central mixing zone are further colored through $\mathrm{OH}$ mass fractions. The black line indicates the limit of Infinitely Fast Chemistry (IFC) or chemical equilibrium, which has been calculated as adiabatic equilibrium calculation using CANTERA ${ }^{45]}$ It represents states where the chemical time scales are considerably shorter than the time scales of mixing. A large share of points in the flame zone (indicated by high $\mathrm{OH}$ mass fractions) is in the vicinity of this line, close to maximum temperatures and near the stoichiometric mixture fraction. This suggests that these states are controlled by mixing and evaporation processes, rather than chemical kinetics ${ }^{33}$ Since the overall system is exposed to thermal losses due to the isothermal boundary conditions, the IFC boundary is not reached in the simulation. Points of the the corner recirculation zones and the upper mixing zone are located on narrow bands near the chemical equilibrium line and above temperatures of $1000 \mathrm{~K}$ and $1500 \mathrm{~K}$, respectively. Points far beyond the IFC boundary denote slow chemistry states, in which mixing time scales may be significantly shorter than chemical time scales and where both fuel and oxidizer can coexist. $\frac{33}{3}$ These points correspond almost entirely to the central mixing zone, due to the interaction between recirculated hot exhaust gases and incoming fresh air. The broad spreading of points among the entire mixture fraction space of $\xi \in\left[0, \xi_{s t}\right]$ indicates, that a large share of states in the combustion chamber is influenced by FRC effects. Thus, the FRC approach seems to be necessary to enable a detailed analysis of the burners flame zone.

As mentioned in the work of Jones et al., $\frac{6}{6}$ the investigated burner offers a complex flame structure with a coexistence of premixed combustion between air and vapor created from the droplet evaporation process, and non-premixed combustion in the vicinity of fuel droplets encountering regions of hot exhaust gases. This is further analyzed in this work by means of the Takeno flame index $\stackrel{46}{36}$ The employed flame index is based

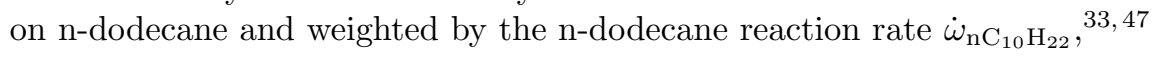

$$
\Theta=\left|\dot{\omega}_{\mathrm{nC}_{10} \mathrm{H}_{22}}\right| \frac{\nabla Y_{\mathrm{nC}_{10} \mathrm{H}_{22}} \cdot \nabla Y_{\mathrm{O}_{2}}}{\left|\nabla Y_{\mathrm{nC}_{10} \mathrm{H}_{22}} \cdot \nabla Y_{\mathrm{O}_{2}}\right|} .
$$

Fig. 15b shows the calculated flame index $\Theta$ in the combustion chamber by colored lines, as well as the magnitued of the temperature gradient in grey contours. The flame index is limited to absolute values of $|\Theta|<50$, in order to clarify the different flame regions. Positive flame indices, indicating premixed regions, appear predominantly as coherent structures near large temperature gradients, which correspond to the flame front. Negative flame indices, which correspond to diffusion flames, occur particularly isolated and are less correlated to the temperature gradient. Comparing both Figs. 15a and 15b, diffusion-driven flames tend to arise in regions of high mixture fractions. The greatest part of the flame is dominated by premixed regions, leading to the assumption that most droplets fully evaporate before they reach the reaction zone. The respective thermo-chemical state space is presented in Fig. 15d, with the states colored by respective flame indices. Points with low flame indices $|\Theta|<10$ are not shown for reasons of clarity. Negative flame indices (diffusion) are located mainly near the IFC boundary at temperatures above $1500 \mathrm{~K}$. Contrary, positive indices (premixed) are also present in the slow chemistry region, which relates to mixing driven regions. This further supports the utilization of the FRC approach, since the flame is cleary influenced by non-eqillibrium premixed flame states. 


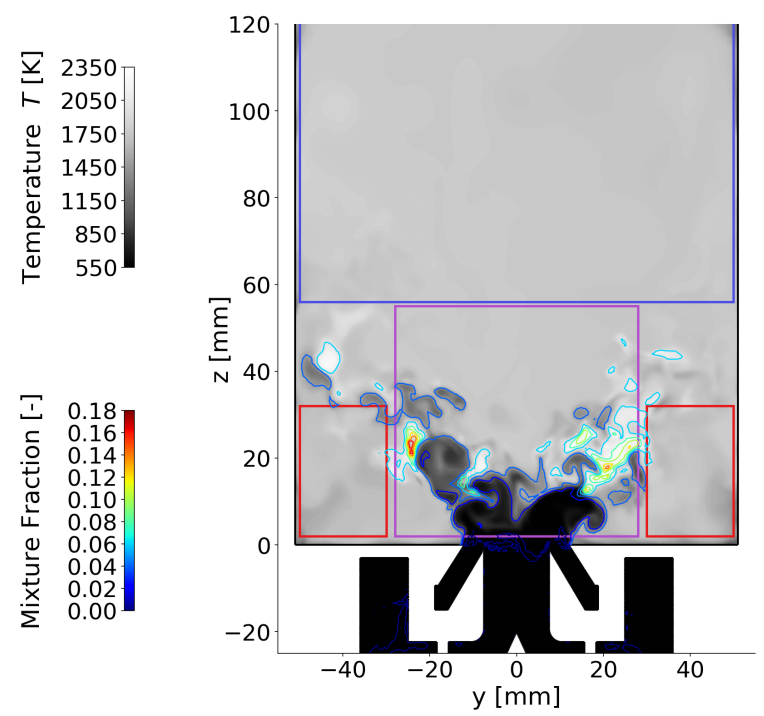

(a) Distribution of temperature and mixture fraction in the $y z$-plane. The colored rectangles denote the central mixing zone, the external recirculating zones and the upper mixing zone, that are mentioned in Fig. $15 \mathrm{c}$

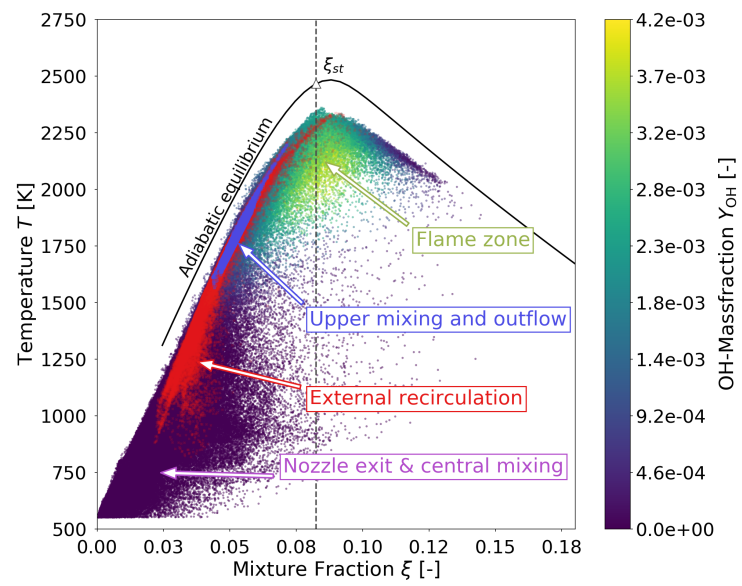

(c) Thermo-chemical state space for different regions in the combustion chamber, which are indicated in Fig. 15a

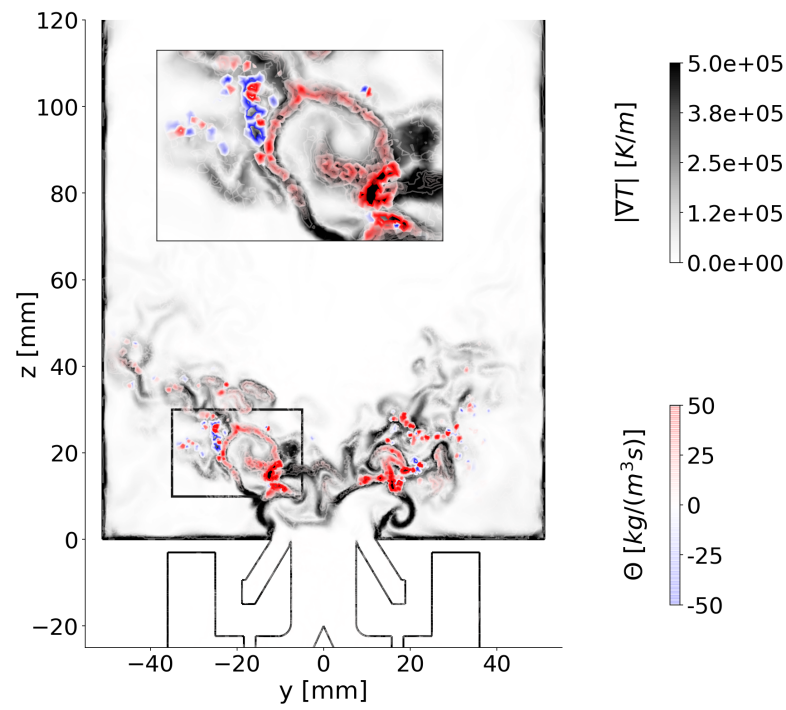

(b) Distribution of the temperature gradient and the Takeno flame index in the $y z$-plane

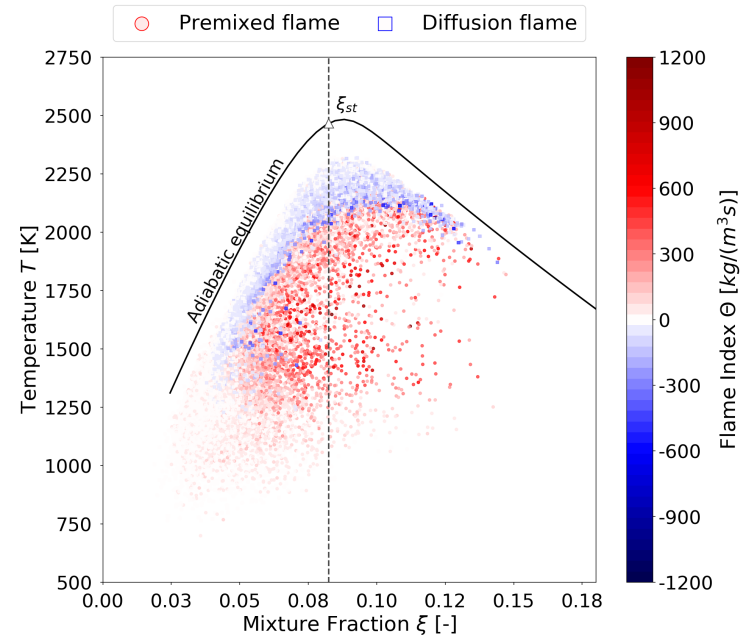

(d) Thermo-chemical state space, restricted to the Takeno flame index.

Figure 15: Instantaneous distribution of different flow properties in the combustion chamber with respective thermo-chemical state spaces.

\section{Conclusion}

LES have been performed for the DLR Generic Single Sector Combustor. The setup consists of a reactive multi-phase flow in a complex geometry, since the burner features a co-rotating double radial swirler and liquid fuel injection through a pre-filming air blast atomizer. Three different meshes were investigated concerning the influence of mesh resolution properties for a non-reactive operating point. Averaged and RMS velocity profiles of all three simulations showed a good agreement with experimental LDA profiles. A detailed chemistry approach for Jet-A1 combustion has been employed for the reactive operating point, combined with a Lagrangian particle method for the disperse fuel phase. Physical properties of the liquid phase were calculated by means of a continuous thermodynamics approach and a multi-component vaporization model was utilized. Concerning the liquid fuel distribution, droplet velocities and SMDs, the applied spray boundary 
conditions showed good agreement to experimental measurements. The shape and the lift-off height of the reaction zone could also be captured well in the LES results. Apart from uncertainties associated with the experimental temperature measurements, discrepancies were obtained for the averaged temperature field in terms of absolute values and locations of high temperature lobes in the vicinity of the reaction zone. The averaged LES results also revealed, that the temperature field was not yet statistically fully converged. The analysis of instantaneous results showed the potential of high fidelity LES to gain insight in the combustion process of turbulent spray flames. Through the utilization of the FRC approach, geometric locations in the combustion chamber could be related to regions in the thermo-chemical state space. Premixed and diffusion-dominated flame regions were determined by means of the Takeno flame index, whereas the burner offered a predominantely premixed behaviour. Furthermore, premixed flame zones were determined to occur in coherent structures, mainly in areas of high temperature gradients. Diffusion flame regions were identified predominantly as isolated spots of high mixture fractions. The FRC approach employed was found to be beneficial for the simulation of the DLR Generic Single Sector Combustor, since it was exposed that premixed flame states appeared in mixing controlled regions, where FRC effects are dominating.

\section{Acknowledgements}

The results were obtained through funding from the DLR-internal project SkaMa-SLM (Skalierte Magerbrennkammer in SLM-Bauweise). Furthermore, the authors would like to thank Dr. G. Eckel and Dr. A. Fiolitakis for their support with the numerical simulations.

\section{References}

\footnotetext{
1 "European Comission, Flightpath 2050, Europe's Vision for Aviation - Report of the High Level Group on Aviation Research," 2011.

${ }^{2}$ Dunn-Rankin, D., Lean Combustion: Technology and Control, Academic Press, 2011.

${ }^{3}$ Gicquel, L. Y., Staffelbach, G., and Poinsot, T., "Large Eddy Simulations of Gaseous Flames in Gas Turbine Combustion Chambers," Progress in Energy and Combustion Science, Vol. 38, No. 6, 2012, pp. 782-817.

${ }^{4}$ Pope, S. B., Turbulent Flows, Cambridge University Press, 2000.

${ }^{5}$ Fröhlich, J., Large Eddy Simulation turbulenter Strömungen, Vol. 1, Springer, 2006.

${ }^{6}$ Jones, W., Marquis, A., and Vogiatzaki, K., "Large-Eddy Simulation of Spray Combustion in a Gas Turbine Combustor," Combustion and Flame, Vol. 161, No. 1, 2014, pp. 222-239.

${ }^{7}$ Andreini, A., Bertini, D., Mazzei, L., and Puggelli, S., "Assessment of scale resolved CFD methods for the investigation of lean burn spray flames," ASME Turbo Expo 2016: Turbomachinery Technical Conference and Exposition, American Society of Mechanical Engineers, 2016.

${ }^{8}$ Puggelli, S., Paccati, S., Bertini, D., Mazzei, L., Giusti, A., and Andreini, A., "Multi-Coupled Numerical Simulations of the DLR Generic Single Sector Combustor," Combustion Science and Technology, 2018, pp. 1-17.

${ }^{9}$ Puggelli, S., Bertini, D., Mazzei, L., and Andreini, A., "Modeling Strategies for Large Eddy Simulation of Lean Burn Spray Flames," Journal of Engineering for Gas Turbines and Power, Vol. 140, No. 5, 2018, pp. 051501.

${ }^{10}$ Meier, U., Heinze, J., Freitag, S., and Hassa, C., "Spray and Flame Structure of a Generic Injector at Aeroengine Conditions," Journal of Engineering for Gas Turbines and Power, Vol. 134, No. 3, 2012, pp. 031503.

${ }^{11}$ Freitag, S., Meier, U., Heinze, J., Behrendt, T., and Hassa, C., "Measurement of Initial Conditions of a Kerosene Spray from a Generic Aeroengine Injector at Elevated Pressure," Atomization and Sprays, Vol. 21, No. 6, 2011.

${ }^{12}$ Knopp, T., Zhang, X., Kessler, R., and Lube, G., "Enhancement of an Industrial Finite-Volume Code for Large-EddyType Simulation of Incompressible High Reynolds Number Flow using Near-Wall Modelling," Computer Methods in Applied Mechanics and Engineering, Vol. 199, No. 13-16, 2010, pp. 890-902.

${ }^{13}$ Le Clercq, P., Doué, N., Rachner, M., and Aigner, M., "Validation of a Multicomponent-Fuel Model for Spray Computations," 47th AIAA Aerospace Sciences Meeting including The New Horizons Forum and Aerospace Exposition, 2009, p. 1188.

${ }^{14}$ Garnier, E., Adams, N., and Sagaut, P., Large Eddy Simulation for Compressible Flows, Springer Science \& Business Media, 2009.

${ }^{15}$ Löwe, J., Probst, A., Knopp, T., and Kessler, R., "A Low-Dissipation Low-Dispersion Second-Order Scheme for Unstructured Finite-Volume Flow Solvers," 53rd AIAA Aerospace Sciences Meeting, 2015, p. 0815.

${ }^{16}$ Probst, A., Löwe, J., Reuss, S., Knopp, T., and Kessler, R., "Scale-Resolving Simulations with a Low-Dissipation LowDispersion Second-Order Scheme for Unstructured Finite-Volume Flow Solvers," 53rd AIAA Aerospace Sciences Meeting, 2015, p. 0816.

${ }^{17}$ Hirsch, C., Numerical Computation of Internal and External Flows: Computational Methods for Inviscid and Viscous Flows, Vol. 1 and 2, John Wiley \& Sons, Chichester, New York, 1990.

${ }^{18}$ Gerlinger, P., Numerische Verbrennungssimulation: Effiziente numerische Simulation turbulenter Verbrennung, Springer-Verlag, 2005.

${ }^{19}$ Ducros, F., Nicoud, F., and Poinsot, T., "Wall-Adapting Local Eddy-Viscosity Models for Simulations in Complex Geometries," Numerical Methods for Fluid Dynamics VI, 1998, pp. 293-299.
} 
${ }^{20}$ Nicoud, F. and Ducros, F., "Subgrid-Scale Stress Modelling Based on the Square of the Velocity Gradient Tensor," Flow, turbulence and Combustion, Vol. 62, No. 3, 1999, pp. 183-200.

${ }^{21}$ Kuo, K. K., Principles of Combustion, Wiley-Interscience, 1986.

${ }^{22}$ Gerlinger, P., "Investigation of an Assumed PDF Approach for Finite-Rate Chemistry," Combustion Science and Technology, Vol. 175, No. 5, 2003, pp. 841-872.

${ }^{23}$ Gerlinger, P., Möbus, H., and Brüggemann, D., "An Implicit Multigrid Method for Turbulent Combustion," Journal of Computational Physics, Vol. 167, No. 2, 2001, pp. 247-276.

${ }^{24}$ Domenico, M. D., Numerical Simulations of Soot Formation in Turbulent Flows, Ph.D. thesis, Fakultät für Luft- und Raumfahrttechnik und Geodäsie, Universität Stuttgart, 2008.

${ }^{25}$ Girimaji, S., "Assumed $\beta$-pdf Model for Turbulent Mixing: Validation and Extension to Multiple Scalar Mixing," Combustion Science and Technology, Vol. 78, No. 4-6, 1991, pp. 177-196.

${ }^{26}$ Baurle, R. and Girimaji, S., "Assumed PDF Turbulence-Chemistry Closure with Temperature-Composition Correlations," Combustion and Flame, Vol. 134, No. 1-2, 2003, pp. 131-148.

${ }^{27}$ Slavinskaya, N. and Frank, P., "A Modelling Study of Aromatic Soot Precursors Formation in Laminar Methane and Ethene Flames," Combustion and flame, Vol. 156, No. 9, 2009, pp. 1705-1722.

${ }^{28}$ Slavinskaya, N. and Haidn, O., "Reduced Chemical Model for High Presure Methane Combustion with PAH Formation," 46th AIAA Aerospace Sciences Meeting and Exhibit, 2008, p. 1012.

${ }^{29}$ Slavinskaja, N., "Skeletal Mechanism for Kerosene Combustion with PAH Production," 46th AIAA Aerospace Sciences Meeting and Exhibit, 2008, p. 992.

${ }^{30}$ Blacha, T., Di Domenico, M., Gerlinger, P., and Aigner, M., "Soot Predictions in Premixed and Non-Premixed Laminar Flames using a Sectional Approach for PAHs and Soot," Combustion and Flame, Vol. 159, No. 1, 2012, pp. $181-193$.

${ }^{31}$ Blacha, T., Di Domenico, M., Slavinskaya, N., Gerlinger, P., and Aigner, M., "Modeling of Soot Size Distributions in Partially Premixed C2H4/Air Flames," 49th AIAA Aerospace Sciences Meeting including the New Horizons Forum and Aerospace Exposition, 2011, p. 414.

${ }^{32}$ Blacha, T., Di Domenico, M., Köhler, M., Gerlinger, P., and Aigner, M., "Soot Modeling in a Turbulent Unconfined $\mathrm{C} 2 \mathrm{H} 4 /$ Air Jet Flame," 49th AIAA aerospace sciences meeting including the new horizons forum and aerospace exposition, 2011, p. 114.

${ }^{33}$ Eckel, G., Grohmann, J., Cantu, L., Slavinskaya, N., Kathrotia, T., Rachner, M., Le Clercq, P., Meier, W., and Aigner, M., "LES of a Swirl-Stabilized Kerosene Spray Flame with a Multi-Component Vaporization Model and Detailed Chemistry," Combustion and Flame, Vol. 207, 2019, pp. 134-152.

${ }^{34}$ Eckel, G., Large Eddy Simulation of Turbulent Reacting Multi-Phase Flows, Ph.D. thesis, Fakultät für Luft- und Raumfahrttechnik und Geodäsie, Universität Stuttgart, 2018.

${ }^{35}$ Eckel, G., Le Clercq, P., Kathrotia, T., Saenger, A., Fleck, S., Mancini, M., Kolb, T., and Aigner, M., "Entrained Flow Gasification. Part 3: Insight into the Injector Near-Field by Large Eddy Simulation with Detailed Chemistry," Fuel, Vol. 223, 2018, pp. 164-178.

${ }^{36}$ Bini, M. and Jones, W. P., "Large-Eddy Simulation of Particle-Laden Turbulent Flows," Journal of Fluid Mechanics, Vol. 614, 2008, pp. 207-252.

${ }^{37}$ Tanner, F., "Development and Validation of a Cascade Atomization and Drop Breakup Model for High-Velocity Dense Sprays," Atomization and Sprays, Vol. 14, No. 3, 2004.

${ }^{38}$ Abramzon, B. and Sirignano, W., "Droplet Vaporization Model for Spray Combustion Calculations," International Journal of Heat and Mass Transfer, Vol. 32, No. 9, 1989, pp. $1605-1618$.

${ }^{39}$ Doué, N., Le Clercq, P., and Aigner, M., "Validation of a Multicomponent-Fuel Droplet Evaporation Model Based on Continuous Thermodynamics," Proceeding of ICLASS 2006, 2006.

${ }^{40}$ Liu, Z. and Phillips, J. B., "Comprehensive Two-Dimensional Gas Chromatography using an On-Column Thermal Modulator Interface," Journal of Chromatographic Science, Vol. 29, No. 6, 06 1991, pp. 227-231.

${ }^{41}$ Syred, N., "A Review of Oscillation Mechanisms and the Role of the Precessing Vortex Core (PVC) in Swirl Combustion Systems," Progress in Energy and Combustion Science, Vol. 32, No. 2, 2006, pp. 93-161.

${ }^{42}$ Syred, N. and Beer, J., "Combustion in Swirling Flows: A Review," Combustion and Flame, Vol. 23, No. 2, 1974, pp. 143-201.

${ }^{43}$ Huang, Y. and Yang, V., "Dynamics and Stability of Lean-Premixed Swirl-Stabilized Combustion," Progress in Energy and Combustion Science, Vol. 35, No. 4, 2009, pp. 293-364.

${ }^{44}$ Bilger, R., Stårner, S., and Kee, R., "On Reduced Mechanisms for Methane-Air Combustion in Nonpremixed Flames," Combustion and Flame, Vol. 80, No. 2, 1990, pp. 135-149.

${ }^{45}$ Goodwin, D. G., Moffat, H. K., and Speth, R. L., "Cantera: An Object-oriented Software Toolkit for Chemical Kinetics, Thermodynamics, and Transport Processes," http://www.cantera.org, 2017, Version 2.3.0.

${ }^{46}$ Yamashita, H., Shimada, M., and Takeno, T., "A Numerical Study on Flame Stability at the Transition Point of Jet Diffusion Flames," Symposium (International) on Combustion, Vol. 26, Elsevier, 1996, pp. 27-34.

${ }^{47}$ Patel, N. and Menon, S., "Simulation of Spray-Turbulence-Flame Interactions in a Lean Direct Injection Combustor," Combustion and Flame, Vol. 153, No. 1-2, 2008, pp. 228-257. 